\title{
A DIRECT BOUNDARY ELEMENT FORMULATION FOR THE FIRST PLANE PROBLEM IN THE DUAL SYSTEM OF MICROPOLAR ELASTICITY
}

\author{
György Szeidl*, Judit Dudra** \\ * Department of Mechanics, University of Miskolc \\ 3515 Miskolc-Egyetemváros, Hungary \\ gyorgy.szeidlduni-miskolc.hu \\ ** Institute of Logistics and Production Systems \\ H-3519 Miskolctapolca, Iglói út 2. Hungary \\ judit.dudra@gmail.com
}

\begin{abstract}
This paper studies the plane strain problem of micropolar elastostatics assuming that the governing equations are given in terms of stress functions of order one. We clarify the conditions of single valuedness and construct the fundamental solution for the dual basic equations. We then establish the integral equations of the direct method. Numerical examples illustrate the applicability of these integral equations.
\end{abstract}

\section{Introduction}

In their book [1], the COSSERAT brothers assume that the motion of a material particle is described by a displacement field and an independent rotation field. According to this assumption a material particle of the body behaves as if it were a very small rigid body. Under this assumption a correct description of the interaction on the inner surfaces of a solid body requires the existence not only of force stresses but of couple stresses as well. The corresponding stress and couple stress tensors are, however, not symmetric. The COSSERAT brothers did not deal with the issue of the constitutive equations.

The theory was reinvented among others by ERINGEN and SuHibi [2, 3] and ERINGEN [4] who supplemented the theory with the constitutive equations. Books [5] and [6] by NOWACZKY provide an excellent overview on the linear theory of micropolar elasticity

According to the famous TONTI scheme $[7,8]$ the variables in the equations of mathematical physics are categorized as fundamental variables, intermediate variables of the first and second kind and source variables. Problems of mathematical physics can be set up in a primal formulation and in a dual one. In [a primal] $\{$ a dual $\}$ formulation the set of field equations involve the defining equations, which relate the fundamental variables to the intermediate variables of the first kind, the constitutive equations, which connect the intermediate variables of the second kind to those of the first kind, and the balance equations, which relate the intermediate variables of the second kind to the source variables. The intermediate variables of the second kind in the [primal] \{dual\} formulation (system) coincide with the intermediate variables of the first kind in the [dual]\{primal\} formulation (system). In addition the [primal] \{dual \} defining equations identically satisfy the [dual] \{primal $\}$ balance equations.

As regards the primal formulation in micropolar elasticity the displacement and rotation vectors (together are referred to as displacements) are the fundamental variables, the asymmetric strain tensor and the curvature twist tensor (together strains) are the intermediate variables of the first kind while the asymmetric force stresses and couple stresses (together stresses) are the intermediate variables of the 
second kind. The body forces and couples constitute the source variables $[9,10]$. Problems in the primal formulation are governed by the kinematic equations (defining equations), which give the asymmetric strain tensor and curvature twist tensor (together the strains) in terms of the displacement and rotation vectors (together the displacements), HOOKE's law which connects the force and couple stress tensors to the two strain tensors, and the equilibrium equations (the balance equations) which relate the force and couple stresses to the body forces and couples (the source variables).

General solutions to the primal equilibrium equations in terms of stress functions of order one have been established independently of each other by SCHAEFFER [11, 12] and CARLSON [13]. It is worth mentioning that their solutions are equivalent to each other.

For the dual formulation of micropolar elasticity stress functions of order one are the fundamental variables, the force and couple stresses are the intermediate variables of the first kind, the asymmetric strain tensor and the curvature twist tensor are the intermediate variables of the second kind and the tensors of incompatibility (they are equal to zero in real problems) constitute the source variables. Problems in the dual formulation are governed by the representation of stresses in terms of stress functions of order one (dual kinematic or defining equations), the inverse form of HOOKE's law (dual constitutive equations), which relates the dual intermediate variables of the second kind (the strains) to that of the first kind (to the stresses), and the compatibility equations (dual balance equations).

The first plane problem of micropolar elasticity was formulated in the primal system by $[14,5]$. Assuming isotropy, Iesan derived the associated integral equations [15]. Schiavone made these results more accurate and precise for outer regions, and also investigated mixed boundary value problems in [16]. Fuang and Liang [17] developed a boundary element analysis of stress concentration in a micropolar plate to determine the stress concentration factors for some cases.

A dual formulation for the first plane problem was published in paper [18]. To the best of our knowledge, the direct boundary element formulation for the first plane problem in the dual system has not yet been developed. In unpublished work, SZEIDL and IVAN [19] dealt with the formulation of indirect boundary integral equations and presented some existence theorems.

In this paper, we first investigate the conditions of single valuedness for the first plane problem of micropolar elasticity. Using these conditions we develop the fundamental solutions of order one and two. Then we derive the SomigLiana relations both for inner and outer regions. Finally we set up a direct boundary element formulation and present an algorithm for numerical solutions.

The paper is organized into ten sections. Section 2 presents some preliminaries, and Section 3 clarifies the supplementary conditions of single valuedness. The basic equations and the fundamental solutions of order one are established in Section 4. Section 5 deals with the determination of the fundamental solutions of order two. The dual SomigLianA relations for inner and outer regions are derived in Sections 6 and 7. Section 8 clarifies how to determine the stresses on the boundary. The last two sections present some numerical examples and a conclusion. The paper is closed by an Appendix which collects longer proofs and derivations.

\section{Preliminaries}

Throughout this paper $x_{1}=x, x_{2}=y$ and $x_{3}=z$ are rectangular Cartesian coordinates with origin $O$. The coordinate plane $\left(x_{1}, x_{2}\right)$ coincides with the plane of strains. The ordered pair $\left(x_{1}, x_{2}\right)$ is denoted by $x$. \{Greek\}[Latin subscripts] are assumed to have the range $\{(1,2)\}[(1,2,3)]$, and summation over repeated subscripts is implied unless explicitly suspended. The triple connected plane region under consideration (Figure 1) is denoted by $A^{+}$- inner region - and is bounded by the outer contour

$$
\mathcal{L}_{0}=\mathcal{L}_{t 1} \cup \mathcal{L}_{u 2} \cup \mathcal{L}_{t 3} \cup \mathcal{L}_{u 4}
$$

and the inner contours

$$
\mathcal{L}_{1}=\mathcal{L}_{t 5} \quad \text { and } \quad \mathcal{L}_{2}=\mathcal{L}_{u 6}
$$


which - partly or wholly - consists of the $\operatorname{arcs} \mathcal{L}_{t 1}, \mathcal{L}_{t 3}, \mathcal{L}_{t 5}$ and $\mathcal{L}_{u 2}, \mathcal{L}_{u 4}, \mathcal{L}_{u 6}$. Here the first $\{t\}[u]$ in the subscripts expresses that \{displacements [tractions] are prescribed on the arc considered. The second subscript counts the arcs which constitute the contours. Note that the inner contours $\mathcal{L}_{1}$ and $\mathcal{L}_{2}$ lie wholly in the interior of the outer contour $\mathcal{L}_{0}$ and they have no points in common. We assume that each contour has a continuously turning unit tangent $\tau_{\kappa}$ and admits a nonsingular parametrization in terms of its arc length $s$. The outer normal is denoted by $n_{\pi}$. Let $\delta_{\kappa \lambda}$ denote the Kronecker symbol, $\partial_{\alpha}$ the derivatives with respect to $x_{\alpha}$ and $\epsilon_{3 \kappa \lambda}$ the permutation symbol.

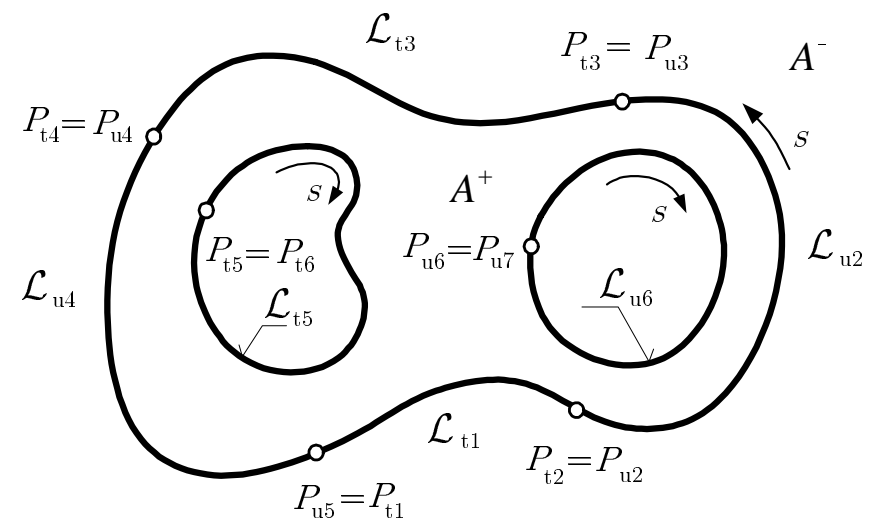

Figure 1:

Let $u_{\kappa}$ and $\varphi_{3}$ be the displacement and microrotation vectors (together the displacements); $\gamma_{\pi \rho}$ and $\kappa_{\rho 3}$ the asymmetric strain tensor and curvature twist tensor (together the strains); and $t_{\pi \rho}$ and $\mu_{\nu 3}, \mu_{3 \nu}$ the asymmetric stress tensor and couple-stress tensor (together the stresses). Body forces and couples are denoted by $b_{\rho}$ and $c_{3}$. Displacements and strains are assumed to be small. \{The symmetric\} [The skew] part of a tensor, say the tensor $t_{\kappa \lambda}$, is denoted by $\left\{t_{(k \lambda)}\right\}\left[t_{<\kappa \lambda>}\right]$.

In the primal system the first plane problem is governed by

- the kinematic equations

$$
\kappa_{\rho 3}-\varphi_{3} \partial_{\rho}=0, \quad \gamma_{\pi \rho}-u_{\rho} \partial_{\pi}-\epsilon_{\rho \pi 3} \varphi_{3}=0
$$

- Hooke's law

$$
\begin{gathered}
t_{\kappa \lambda}=2 \mu\left(\gamma_{(\kappa \lambda)}+\frac{\nu}{1-2 \nu} \gamma_{\phi \phi} \delta_{\kappa \lambda}\right)+2 \alpha \gamma_{\langle\kappa \lambda\rangle} \\
\mu_{\nu 3}=(\gamma+\varepsilon) \kappa_{\nu 3}, \quad \mu_{3 \nu}=(\gamma-\varepsilon) \kappa_{\nu 3}
\end{gathered}
$$

where $\mu, \alpha, \gamma, \nu$ and $\varepsilon$ are the material constants of an isotropic body, and

- the primal balance equations

$$
\partial_{\nu} t_{\nu \rho}+b_{\rho}=0, \quad \partial_{\nu} \mu_{\nu 3}+\epsilon_{3 \nu \rho} t_{\nu \rho}+c_{3}=0 .
$$

Field equations (1), (2a,b) and (3) should be associated with appropriate boundary conditions. If a contour is not divided into parts then either tractions or displacements can be imposed on it. If a contour is divided into parts then it is assumed to consist of an even number of arcs, with alternating prescriptions of displacements and tractions. For the inner region shown in Figure 1 \{tractions\}[displacements] are given on the arc $\left\{\mathcal{L}_{t}=\mathcal{L}_{t 1} \cup \mathcal{L}_{t 3} \cup \mathcal{L}_{t 5}\right\}\left[\mathcal{L}_{u}=\mathcal{L}_{u 2} \cup \mathcal{L}_{u 4} \cup \mathcal{L}_{u 6}\right]$. Letters with hats stand for the prescribed values. The displacement and traction boundary conditions are given by

$$
u_{\kappa}=\hat{u}_{\kappa}, \quad \varphi_{3}=\hat{\varphi}_{3}
$$


and

$$
n_{\nu} t_{\nu \rho}=\hat{t}_{\rho,} \quad n_{\nu} \mu_{\nu 3}=\hat{\mu} .
$$

Field equations (1), (2a,b) and (3) should be associated with appropriate boundary conditions. If a contour is not divided into parts then either tractions or displacements can be imposed on it. If a contour is divided into parts then it is assumed to consist of an even number of arcs, with alternating prescriptions of displacements and tractions. For the inner region shown in Figure 1 \{tractions\}[displacements] are given on the arc $\left\{\mathcal{L}_{t}=\mathcal{L}_{t 1} \cup \mathcal{L}_{t 3} \cup \mathcal{L}_{t 5}\right\}\left[\mathcal{L}_{u}=\mathcal{L}_{u 2} \cup \mathcal{L}_{u 4} \cup \mathcal{L}_{u 6}\right]$. Letters with hats stand for the prescribed values. The displacement and traction boundary conditions are given by

$$
u_{\kappa}=\hat{u}_{\kappa}, \quad \varphi_{3}=\hat{\varphi}_{3}
$$

and

$$
n_{\nu} t_{\nu \rho}=\hat{t}_{\rho,} \quad n_{\nu} \mu_{\nu 3}=\hat{\mu} .
$$

In the dual system the first plane problem of micropolar elastostatics [20] is governed by

- the dual kinematic equations

$$
\begin{gathered}
t_{\pi \rho}=\epsilon_{\pi \mu 3} \partial_{\mu} \mathcal{F}_{\rho}+\stackrel{o}{t}{ }_{\pi \rho}, \\
\mu_{\nu 3}=\epsilon_{\nu \pi 3}\left(\partial_{\pi} \mathcal{H}+\epsilon_{3 \pi \rho} \mathcal{F}_{\rho}\right)+\stackrel{o}{\mu}{ }_{\nu 3}
\end{gathered}
$$

which give the stresses $t_{\pi \rho}$ and $\mu_{\nu 3}$ in terms of stress functions of order one $\mathcal{F}_{\rho}$ and $\mathcal{H}$ while $t_{\pi \rho}^{o}$ and $\stackrel{o}{\mu}_{\nu 3}$ are particular solutions of the primal equilibrium equations,

- the inverse form of HoOKE's law (dual constitutive equations)

$$
\begin{gathered}
\gamma_{\kappa \lambda}=\frac{1}{2 \mu} t_{(\kappa \lambda)}+\frac{1}{2 \alpha} t_{\langle\kappa \lambda\rangle}-\frac{\nu}{2 \mu} t_{\phi \phi} \delta_{\kappa \lambda}, \\
\kappa_{\nu 3}=\frac{1}{\gamma+\varepsilon} \mu_{\nu 3}
\end{gathered}
$$

- and the compatibility differential equations (dual balance equations)

$$
E=\epsilon_{3 \pi \rho} \kappa_{\rho 3} \partial_{\pi}=0 \quad D_{\rho}=\epsilon_{3 \nu \pi}\left(\gamma_{\pi \rho} \partial_{\nu}+\epsilon_{\pi \rho 3} \kappa_{\nu 3}\right)=0 .
$$

REMARK 1.: If $\mu>0, \gamma+\varepsilon>0, \alpha>0, \nu \in(0,0.5)$ then the strain energy density

$$
e\left(t_{11}, \ldots, \mu_{23}\right)=\frac{1}{2}\left\{\frac{1}{2 \mu}\left(t_{(\kappa \lambda)} t_{(\kappa \lambda)}-\nu t_{\varphi \varphi} t_{\rho \rho}\right)+\frac{1}{4 \alpha} t_{<\kappa \lambda>} t_{<\kappa \lambda>}+\frac{1}{\gamma+\varepsilon} \mu_{\rho 3} \mu_{\rho 3}\right\}
$$

is strictly positive provided that at least one stress component is different from zero.

REMARK 2.: For plane problems there are four independent material constants: $\mu, \nu, \alpha$ and $\gamma+\epsilon$. It is customary to introduce the characteristic lengths $\mathfrak{l}, l_{1}$ and the coupling number $N$ which are defined by

$$
\mathfrak{l}^{2}=\frac{\gamma+\epsilon}{4 \mu}, \quad l_{1}^{2}=\frac{(\gamma+\epsilon)(\mu+\alpha)}{4 \mu \alpha}, \quad N^{2}=\frac{\mathfrak{l}^{2}}{l_{1}^{2}}=\frac{\alpha}{\mu+\alpha} .
$$

If $\mathfrak{l}$ and $l_{1}$ tend to zero, the equations of micropolar elasticity simplify to those of classical elasticity. If $N$ tends to 1 we obtain the equations of the couple stress theory.

REMARK 3.: The stress representations $(6 a, b)$ (the dual kinematic equations) are solutions to the primal equilibrium equations. The particular solutions $\stackrel{o}{t}_{\pi \rho}$ and $\stackrel{o}{\mu}_{\nu 3}$ have the form

$$
\stackrel{o}{t_{\pi \rho}}=\partial_{\pi} p_{\rho}, \quad \stackrel{o}{\mu}, \quad=\epsilon_{\nu 3 \eta} p_{\eta}+\partial_{\pi} q
$$


in which

$$
\triangle p_{\rho}=-b_{\rho}, \quad \triangle q_{3}=c_{3} .
$$

These representations were found found independently of each other by SCHAFFER [12] and CARLSON [13].

REMARK 4.: There belong no stresses to the stress functions

$$
\mathcal{F}_{\rho}=\stackrel{o}{\mathcal{F}}_{\rho} \quad \text { and } \quad \mathcal{H}=\stackrel{o}{\mathcal{H}}-\varepsilon_{3 \pi \rho} x_{\pi} \stackrel{o}{\mathcal{F}}_{\rho}
$$

where $\stackrel{o}{\mathcal{F}}_{\rho}$ and $\stackrel{o}{\mathcal{H}}$ are arbitrary constants. These stress functions correspond to the rigid body motion which causes no strains in the region under consideration and are referred to as dual rigid body motion.

REMARK 5.: There are no strains due to the rigid body motion

$$
u_{\rho}=\stackrel{o}{u}_{\rho}+\varepsilon_{3 \pi \rho} x_{\pi} \stackrel{o}{\varphi} \text { and } \quad \varphi_{3}=\stackrel{o}{\varphi}_{3}
$$

where $\stackrel{o}{u}_{\rho}$ and $\stackrel{o}{\varphi}_{3}$ are arbitrary constants. These displacements correspond to those stress functions causing no stresses in the region under consideration.

REMARK 6.: The primal kinematic equations (1) are solutions to the dual balance equations (8).

REMARK 7.: If the compatibility differential equations or, equivalently, the dual balance equations are satisfied, then the strains $\gamma_{\pi \rho}$ and $\kappa_{\nu 3}$ are compatible on a single connected domain, i.e. the primal kinematic equations have one solution for the displacements $u_{\rho}$ and $\varphi_{3}$ provided that we disregard rigid body motions.

Field equations $(6 a, b),(7 a, b)$ and $(8)$ should be associated with appropriate boundary conditions. Kozak and Szeidl [21] have shown that the strain boundary conditions

$$
\begin{array}{rlrl}
\tau_{\pi} \gamma_{\pi \rho} & =\frac{d \hat{u}_{\rho}}{d s}+\tau_{\pi} \epsilon_{\rho \pi 3} \hat{\varphi}_{3}, & & s \in \mathcal{L}_{u} \\
\tau_{\pi} \kappa_{\pi 3}=\frac{d \hat{\varphi}_{3}}{d s}, & s \in \mathcal{L}_{u}
\end{array}
$$

correspond to the displacement boundary conditions of the primal system.

Consider now the boundary conditions on arc $\mathcal{L}_{t}$. Using equations (4b) and (6a,b) we have

$$
\begin{array}{lr}
\hat{t}_{\rho}-\stackrel{\circ}{t}_{\rho}=n_{\pi} \epsilon_{\pi \nu 3}\left(\mathcal{F}_{\rho} \partial_{\nu}\right)=\frac{d \mathcal{F}_{\rho}}{d s}, & s \in \mathcal{L}_{t} \\
\hat{\mu}-\stackrel{\circ}{\mu}=n_{\pi} \epsilon_{\pi \nu 3}\left(\mathcal{H} \partial_{\nu}+\epsilon_{3 \nu \rho} \mathcal{F}_{\rho}\right)=\frac{d \mathcal{H}}{d s}-n_{\rho} \mathcal{F}_{\rho}, & s \in \mathcal{L}_{t}
\end{array}
$$

where

$$
\stackrel{\circ}{t}_{\rho}=n_{\sigma}\left(p_{\rho} \partial_{\nu}\right), \quad \stackrel{\circ}{\mu}=n_{\rho}\left(\epsilon_{\rho 3 \eta} p_{\eta}+q \partial_{\rho}\right) .
$$

To solve the differential equation $(14 \mathrm{a})$, let

$$
\hat{\mathcal{F}}_{\rho}(s)=\int_{P_{t i}}^{s}\left[\hat{t}_{\rho}(\sigma)-\stackrel{\circ}{t}_{\rho}(\sigma)\right] d \sigma, \quad s \in \mathcal{L}_{t i}, \quad i=1,3,5,
$$

and let $C_{\rho}(i=1,3,5)$ be constants of integration. Then any solution of (14a) can be written as $(t i)$

$$
\mathcal{F}_{\rho}(s)=\hat{\mathcal{F}}_{\rho}(s)+\underset{(t i)}{C_{\rho}} \quad s \in \mathcal{L}_{t i} \quad i=1,3,5 .
$$

This equation is one of the boundary conditions for the $\operatorname{arc} \mathcal{L}_{t}$.

Let $\hat{\mathcal{H}}(s)$ be a solution to the differential equation

$$
\hat{\mu}-\stackrel{\circ}{\mu}=\frac{d}{d s} \hat{\mathcal{H}}-n_{\rho} \hat{\mathcal{F}}_{\rho}
$$


Integration yields

$$
\hat{\mathcal{H}}(s)=\int_{P_{t i}}^{s}\left[\hat{\mu}(\sigma)-\stackrel{\circ}{\mu}(\sigma)+n_{\rho} \hat{\mathcal{F}}_{3}^{\rho}\right] d \sigma .
$$

Subtracting equation (17) from (14b) and then using (16) we obtain the differential equation

$$
\frac{d}{d s}(\mathcal{H}-\hat{\mathcal{H}})-\underset{(t i)}{n_{\rho} C_{\rho}}=0
$$

which implies the boundary condition

$$
\mathcal{H}(s)=\hat{\mathcal{H}}(s)+\underset{(t i)}{C}-\varepsilon_{3 \kappa \rho}\left[x_{\kappa}(s)-x_{\kappa}\left(P_{t i}\right)\right] \underset{(t i)}{C_{\rho}} \quad s \in \mathcal{L}_{t i} \quad i=1,3,5
$$

where $C$ is a constant of integration.

(ti)

\section{Supplementary compatibility conditions}

The compatibility field equations (8) do not guarantee single valuedness of the displacements if the region is multiply connected or if tractions are prescribed on more than one boundary arc. In this section we derive compatibility conditions for these cases as well from the principle of minimum complementary energy. As is well known, the total complementary energy functional is

$$
K=-\frac{1}{2} \int_{A^{+}}\left(t_{\pi \rho} \gamma_{\pi \rho}+\mu_{\nu 3} \kappa_{\nu 3}\right) d A+\int_{\mathcal{L}_{u}}\left(n_{\pi} t_{\pi \rho} \hat{u}_{\rho}+n_{\nu} \mu_{\nu 3} \hat{\varphi}_{3}\right) d s .
$$

The stationarity condition

$$
\delta K=-\int_{A^{+}}\left(\gamma_{\pi \rho} \delta t_{\pi \rho}+\kappa_{\nu 3} \delta \mu_{\nu 3}\right) d A+\int_{\mathcal{L}_{u}}\left(n_{\pi} \delta t_{\pi \rho} \hat{u}_{\rho}+n_{\nu} \delta \mu_{\nu 3} \hat{\varphi}_{3}\right) d s=0
$$

ensures that the strains $\gamma_{\pi \rho}$ and $\kappa_{\nu 3}$ satisfy the conditions to be kinematically admissible. In the functional (20) $\gamma_{\pi \rho}$ and $\kappa_{\nu 3}$ are given in terms of the stresses $t_{\kappa \lambda}$ and $\mu_{\nu 3}$ via HOOKE's law while the stresses $t_{\kappa \lambda}$ and $\mu_{\nu 3}$ should satisfy the equilibrium equations and the traction boundary conditions. Consequently, the variations of stresses can not be arbitrary but should meet the side conditions

$$
\begin{aligned}
\partial_{\pi} \delta t_{\pi \rho} & =0, & \partial_{\nu} \delta \mu_{\nu 3}+\epsilon_{3 \nu \rho} \delta t_{\nu \rho} & =0, & & x \in A^{+} \\
n_{\pi} \delta t_{\pi \rho} & =0, & n_{\nu} \delta \mu_{\nu 3} & =0, & & s \in \mathcal{L}_{t} .
\end{aligned}
$$

Both (22) and (23) are satisfied if $\delta t_{\pi \rho}$ and $\delta \mu_{\nu 3}$ are expressed with the stress functions and the integration constants introduced in (16) and (19) as follows

$$
\begin{gathered}
\delta t_{\pi \rho}=\epsilon_{\pi \mu 3} \partial_{\mu} \delta \mathcal{F}_{\rho}, \quad \begin{array}{r}
\delta \mu_{\nu 3}=\epsilon_{\nu \pi 3}\left(\partial_{\pi} \delta \mathcal{H}+\epsilon_{3 \pi \rho} \delta \mathcal{F}_{\rho}\right), \quad \\
\delta \mathcal{F}_{\rho}(s)=\underset{(t i)}{\delta C_{\rho},} \quad x \in A^{+}
\end{array} \\
\delta \mathcal{H}(s)=\delta \hat{\mathcal{H}}(s)+\underset{(t i)}{\delta C}-\varepsilon_{3 \kappa \rho}\left[x_{\kappa}(s)-x_{\kappa}\left(P_{t i}\right)\right] \underset{(t i)}{\delta C_{\rho}, \quad i=1,3,5} \quad s \in \mathcal{L}_{t i}
\end{gathered}
$$

Substituting equations (24) into the extremum condition and applying the Green theorem we have

$$
\delta K=\delta K_{A}+\delta K_{\mathcal{L}}+\delta K_{u}=0
$$

where

$$
\delta K_{A}=-\int_{A^{+}}\left[D_{\rho} \delta \mathcal{F}_{\rho}+E \delta \mathcal{H}\right] d A=0
$$


and

$$
\begin{aligned}
\delta K_{\mathcal{L}} & =\int_{\mathcal{L}_{u} \cup \mathcal{L}_{t}}\left(n_{\mu} \epsilon_{\mu \pi 3} \gamma_{\pi \rho} \delta \mathcal{F}_{\rho}+n_{\pi} \epsilon_{\pi \nu 3} \kappa_{\nu 3} \delta \mathcal{H}\right) d s \\
\delta K_{u} & =\int_{\mathcal{L}_{u}}\left[n_{\pi} \epsilon_{\pi \mu 3} \delta \mathcal{F}_{\rho} \partial_{\mu} \hat{u}_{\rho}+n_{\nu} \varepsilon_{\nu \psi 3}\left(\delta \mathcal{H} \partial_{\psi}+\epsilon_{3 \psi \rho} \delta \mathcal{F}_{\rho}\right) \hat{\varphi}_{3}\right] d s .
\end{aligned}
$$

To derive the final expression for $\delta K_{\mathcal{L}}+\delta K_{u}$, we combine the following observations:

- $n_{\pi} \varepsilon_{\pi \mu 3}=\tau_{\mu}$ and $\mathcal{L}=\mathcal{L}_{u} \cup \mathcal{L}_{t}$;

- the variations of stress functions on $\mathcal{L}_{t}$ are given by (24) and (25);

- the variations of stress functions are continuous on $\mathcal{L}$ including the points $P_{t 1}, P_{t 2}, P_{t 3}$ and $P_{t 4}$ as well;

- the desired result can be achieved if we perform partial integrations when transforming $\delta K_{u}$ and take the continuity of the stress functions into account.

Building on these steps, we can integrate by parts the expression for $\delta K_{u}$ and use the continuity of the stress functions to obtain from (28a) and (28b) the following result:

$$
\begin{gathered}
\delta K_{\mathcal{L}}+\delta K_{u}= \\
=\int_{\mathcal{L}_{u}}\left[\tau_{\pi} \gamma_{\pi \rho}-\frac{d \hat{u}_{\rho}}{d s}-\tau_{\pi} \epsilon_{\rho \pi 3} \hat{\varphi}_{3}\right] \delta \mathcal{F}_{\rho} d s+\int_{\mathcal{L}_{u}}\left[\tau_{\pi} \kappa_{\pi 3}-\frac{d \hat{\varphi}_{3}}{d s}\right] \delta \mathcal{H} d s+ \\
+\oint_{\mathcal{L}_{t 5}} \tau_{\pi} \kappa_{\pi 3} d s \delta \underset{(t 5)}{C}+\oint_{\mathcal{L}_{t 5}} \tau_{\pi}\left[\gamma_{\pi \rho}-\kappa_{\pi 3} \epsilon_{\rho 3 \sigma}\left(x_{\sigma}-x_{\sigma}\left(P_{t 5}\right)\right)\right] d s \delta C_{\psi}+ \\
+\sum_{(t 5)}\left\{\int_{\mathcal{L}_{t i}} \tau_{\pi} \kappa_{\pi 3} d s-\left.\hat{\varphi}_{3}\right|_{P_{t i}} ^{P_{t, i+1}}\right\} \delta \underset{(t i)}{C+} \\
+\sum_{i=1,3}\left\{\oint_{\mathcal{L}_{t i}} \tau_{\pi}\left[\gamma_{\pi \rho}-\kappa_{\pi 3} \epsilon_{\rho 3 \sigma}\left(x_{\sigma}-x_{\sigma}\left(P_{t 5}\right)\right)\right] d s-\right. \\
\left.-\left.\hat{u}_{\rho}\right|_{P_{t i}} ^{P_{t, i+1}}+\hat{\varphi}_{3} \epsilon_{\rho 3 \sigma}\left(x_{\sigma}\left(P_{t, i+1}\right)-x_{\sigma}\left(P_{t i}\right)\right)\right\} \underset{(t i)}{\delta C_{\rho} .}
\end{gathered}
$$

Since the variations are arbitrary in (27) and (29), we obtain four groups of equations:

- the compatibility conditions on $A^{+}$:

$$
E(x)=0, \quad D_{\rho}(x)=0 ;
$$

- the strain boundary conditions on $\mathcal{L}_{u}$ :

$$
\tau_{\pi} \gamma_{\pi \rho}-\frac{d \hat{u}_{\rho}}{d s}-\tau_{\pi} \epsilon_{\rho \pi 3} \hat{\varphi}_{3}=0, \quad \tau_{\pi} \kappa_{\pi 3}-\frac{d \hat{\varphi}_{3}}{d s}=0
$$

- the compatibility conditions at large on $\mathcal{L}_{1}$ :

$$
\oint_{\mathcal{L}_{1}} \tau_{\pi}\left[\gamma_{\pi \rho}-\kappa_{\pi 3} \epsilon_{\rho 3 \sigma}\left(x_{\sigma}-x_{\sigma}\left(P_{t 5}\right)\right)\right] d s=0, \quad \oint_{\mathcal{L}_{1}} \tau_{\pi} \kappa_{\pi 3} d s=0 ;
$$

and 
- the supplementary boundary conditions on $\mathcal{L}_{0}$

$$
\begin{gathered}
\oint_{\mathcal{L}_{t i}} \tau_{\pi}\left[\gamma_{\pi \rho}-\kappa_{\pi 3} \epsilon_{\rho 3 \sigma}\left(x_{\sigma}-x_{\sigma}\left(P_{t 5}\right)\right)\right] d s-\left.\hat{u}_{\rho}\right|_{P_{t i}} ^{P_{t, i+1}}+ \\
+\hat{\varphi}_{3} \epsilon_{\rho 3 \sigma}\left(x_{\sigma}\left(P_{t, i+1}\right)-x_{\sigma}\left(P_{t i}\right)\right)=0, \quad i=1,3 \\
\int_{\mathcal{L}_{t i}} \tau_{\pi} \kappa_{\pi 3} d s-\left.\hat{\varphi}_{3}\right|_{P_{t i}} ^{P_{t, i+1}}=0 .
\end{gathered}
$$

REMARK 8.: It can be shown that only two of the three macro conditions of compatibility - the compatibility condition at the large (32) and the supplementary conditions of single valuedness (33a,b) are independent of each other. Since each of these conditions involves three equations, we can set three of the nine undetermined constants - for example, $\underset{(t 1)}{C}$ and $\underset{(t 1)}{\underset{\rho}{\rho}} \rho-$ to zero. This follows because no stresses belong to the corresponding stress functions

$$
\mathcal{F}_{\rho}(s)=\underset{(t i)}{C} \rho \quad \text { and } \quad \mathcal{H}(s)=\underset{(t i)}{C}-\varepsilon_{3 \kappa \rho}\left[x_{\kappa}(s)-x_{\kappa}\left(P_{t i}\right)\right] \underset{(t i)}{\underset{C}{\rho} \rho} .
$$

As a result, the number of independent macro conditions for single valuedness equals the number of undetermined constants of integration.

\section{Basic equations and fundamental solutions of order one}

Here and in the sequel we shall assume that there are no body forces. Substituting the dual kinematic equation $(6 a, b)$ into HoOKE's law $(7 a, b)$ and the result into the compatibility equations $(8)$ we have

$$
\begin{gathered}
{\left[-\left(\frac{1}{4 \mu}+\frac{1}{4 \alpha}\right) \partial_{1} \partial_{1}-\frac{1-\nu}{2 \mu} \partial_{2} \partial_{2}+\frac{1}{\gamma+\varepsilon}\right] \mathcal{F}_{1}+\left(\frac{1}{4 \mu}-\frac{1}{4 \alpha}-\frac{\nu}{2 \mu}\right) \partial_{1} \partial_{2} \mathcal{F}_{2}-} \\
-\frac{1}{\gamma+\varepsilon} \partial_{2} \mathcal{H}=0 \\
\left(\frac{1}{4 \mu}-\frac{1}{4 \alpha}-\frac{\nu}{2 \mu}\right) \partial_{2} \partial_{1} \mathcal{F}_{1}+\left[-\left(\frac{1}{4 \mu}+\frac{1}{4 \alpha}\right) \partial_{2} \partial_{2}-\frac{1-\nu}{2 \mu} \partial_{1} \partial_{1}+\frac{1}{\gamma+\varepsilon}\right] \mathcal{F}_{2} \\
\frac{1}{\gamma+\varepsilon} \partial_{2} \mathcal{F}_{1}-\frac{1}{\gamma+\varepsilon} \partial_{1} \mathcal{F}_{2}-\frac{1}{\gamma+\varepsilon} \Delta \mathcal{H}=0 .
\end{gathered}
$$

The quantities that can be prescribed on the arcs constituting $\mathcal{L}_{u}$ are obtained from equations (13a,b):

$$
\begin{aligned}
& \tau_{1} \gamma_{11}+\tau_{2} \gamma_{21}=\left\{\frac{1-\nu}{2 \mu} \tau_{1} \partial_{2}-\left(\frac{1}{4 \mu}+\frac{1}{4 \alpha}\right) \tau_{1} \partial_{1}\right\} \mathcal{F}_{1}+ \\
& +\left\{\left(\frac{1}{4 \mu}-\frac{1}{4 \alpha}\right) \tau_{2} \partial_{2}+\frac{\nu}{2 \mu} \tau_{1} \partial_{1}\right\} \mathcal{F}_{2} \\
& \tau_{1} \gamma_{12}+\tau_{2} \gamma_{22}=\left\{\left(\frac{1}{4 \mu}-\frac{1}{4 \alpha}\right) \tau_{1} \partial_{1}-\frac{\nu}{2 \mu} \tau_{2} \partial_{2}\right\} \mathcal{F}_{1}+ \\
& +\left\{-\frac{1+\nu}{2 \mu} \tau_{2} \partial_{1}+\left(\frac{1}{4 \mu}+\frac{1}{4 \alpha}\right) \tau_{1} \partial_{2}\right\} \mathcal{F}_{2}
\end{aligned}
$$




$$
\tau_{1} \kappa_{13}+\tau_{2} \kappa_{23}=\frac{1}{\gamma+\varepsilon}\left\{-\tau_{1} \mathcal{F}_{1}-\tau_{2} \mathcal{F}_{2}+\tau_{1} \partial_{2} \mathcal{H}-\tau_{2} \partial_{1} \mathcal{H}\right\}
$$

Let

$$
\mathfrak{a}=\frac{(\gamma+\varepsilon)(1-\nu)}{2 \mu}
$$

be a new constant. It is obvious - see REMARK 1. for details - that $\mathfrak{a}>0$. After multiplying equations (34a,b,c) with $\gamma+\varepsilon$ and straightforward manipulations we arrive at the basic equations in the dual system of the first plane problem:

$$
\begin{aligned}
\left(1-\mathfrak{l}^{2} \Delta\right) \mathcal{F}_{1}+\left(\mathfrak{l}^{2}-\mathfrak{a}\right)\left(\mathcal{F}_{1} \partial_{2}-\mathcal{F}_{2} \partial_{1}\right) \partial_{2}-\partial_{2} \mathcal{H} & =0 \\
\left(\mathfrak{l}^{2}-\mathfrak{a}\right)\left(\mathcal{F}_{1} \partial_{2}-\mathcal{F}_{2} \partial_{1}\right) \partial_{1}+\left(1-\mathfrak{l}^{2} \Delta\right) \mathcal{F}_{2}+\partial_{1} \mathcal{H} & =0 \\
\partial_{2} \mathcal{F}_{1}-\partial_{1} \mathcal{F}_{2}-\Delta \mathcal{H} & =0
\end{aligned}
$$

or in matrix form

$$
\mathfrak{D}_{l k} \mathfrak{u}_{k}=0,
$$

where

$$
\mathfrak{D}_{l k}=\left[\begin{array}{ccc}
1-\mathfrak{l}^{2} \Delta+\left(\mathfrak{l}^{2}-\mathfrak{a}\right) \partial_{2} \partial_{2} & -\left(\mathfrak{l}^{2}-\mathfrak{a}\right) \partial_{1} \partial_{2} & -\partial_{2} \\
-\left(\mathfrak{l}^{2}-\mathfrak{a}\right) \partial_{1} \partial_{2} & 1-\mathfrak{l}^{2} \Delta+\left(\mathfrak{l}^{2}-\mathfrak{a}\right) \partial_{1} \partial_{1} & \partial_{1} \\
\partial_{2} & -\partial_{1} & -\Delta
\end{array}\right]
$$

is the corresponding differential operator and

$$
\mathfrak{u}_{k}=\left(\mathcal{F}_{1}\left|\mathcal{F}_{2}\right| \mathcal{H}\right)
$$

is the vector of unknowns, i.e., the vector of stress functions or the dual displacement field.

REMARK 9.: The system of differential equations (40) is elliptic if the material constants meet our preconditions - see REMARK 1. for details.

Let $D_{k j}$ be the cofactor of $\mathfrak{D}_{j k}$ :

$$
\begin{gathered}
{\left[D_{k l}\right]=} \\
=\left[\begin{array}{ccc}
-\left[1-\mathfrak{l}^{2} \Delta+\left(\mathfrak{l}^{2}-\mathfrak{a}\right) \partial_{1}^{2}\right] \Delta+\partial_{1}^{2} & -\left(\mathfrak{l}^{2}-\mathfrak{a}\right) \Delta \partial_{1} \partial_{2}+\partial_{1} \partial_{2} & \left(1-\mathfrak{l}^{2} \Delta\right) \partial_{2} \\
-\left(\mathfrak{l}^{2}-\mathfrak{a}\right) \Delta \partial_{2} \partial_{1}+\partial_{2} \partial_{1} & -\left[1-l^{2} \Delta+\left(\mathfrak{l}^{2}-\mathfrak{a}\right) \partial_{2}^{2}\right] \Delta+\partial_{2}^{2} & -\left(1-\mathfrak{l}^{2} \Delta\right) \partial_{1} \\
-\left(1-\mathfrak{l}^{2} \Delta\right) \partial_{2} & \left(1-\mathfrak{l}^{2} \Delta\right) \partial_{1} & \left(1-\mathfrak{l}^{2} \Delta\right)(1-\mathfrak{a} \Delta)
\end{array}\right] .
\end{gathered}
$$

It is obvious that

$$
D_{i k} \mathfrak{D}_{k l}=\mathfrak{D}_{i k} D_{k l}=\operatorname{det}\left(\mathfrak{D}_{j l}\right) \delta_{i l}
$$

where

$$
\operatorname{det}\left(\mathfrak{D}_{j l}\right)=\mathfrak{a}\left(1-\mathfrak{l}^{2} \Delta\right) \Delta \Delta
$$

If we introduce a new unknown $\chi_{l}$ [22], [23] defined by the equation

$$
\mathfrak{u}_{k}=D_{k l} \chi_{l}
$$

and substitute it back into equation (22), we obtain an uncoupled system of differential equations

$$
\mathfrak{D}_{i k} \mathfrak{u}_{k}=\mathfrak{D}_{i k} D_{k l} \chi_{l}=\operatorname{det}\left(\mathfrak{D}_{j l}\right) \chi_{i}=0 .
$$

Let $Q\left(\xi_{1}, \xi_{2}\right)$ and $M\left(x_{1}, x_{2}\right)$ be two points in the plane of strain (the source point and the field point). Further let $\mathbf{e}$ with components $e_{i}$ be a unit vector at $Q$. We shall assume temporarily that the point $Q$ is 
fixed. The distance between $Q$ and $M$ is $R$, the position vector of $M$ relative to $Q$ is $r_{\kappa}$. We call the solution of the differential equation

$$
\mathfrak{D}_{l k} \mathfrak{u}_{k}+(\gamma+\varepsilon) \delta(M-Q) e_{l}=0
$$

in which $\delta(M-Q)$ is the Dirac function the fundamental solution. It follows from our earlier arguments see in particular (45), (46) and (48) - that the fundamental solution can be obtained from the fundamental solution for the Galjorkin functions $\chi_{l}$, i.e., from the solution of the differential equation

$$
\mathfrak{D}_{i k} \mathfrak{u}_{k}+(\gamma+\varepsilon) \delta(M-Q) e_{i}=\mathfrak{a}\left(1-\mathfrak{l}^{2} \Delta\right) \Delta \Delta \chi_{i}+(\gamma+\varepsilon) \delta(M-Q) e_{i}=0
$$

Let

$$
k^{2}=\frac{1}{\mathfrak{l}^{2}} \quad \text { and } \quad a=\frac{\gamma+\varepsilon}{\mathfrak{a l}^{2}}=\frac{2 \mu k^{2}}{1-\nu}
$$

be further constants. With this notation, equation (49) implies that

$$
\left(\Delta-k^{2}\right) \Delta \Delta \chi_{i}=a \delta(M-Q) e_{i}
$$

Consequently

$$
\chi_{i}(M, Q)=\chi(R) e_{i} \quad \chi(R)=-\frac{a}{8 \pi k^{4}}\left[k^{2} R^{2} \ln R+4 \ln R+4 K_{o}(k R)\right]
$$

are the solutions for the Galjorkin functions - see [15], [24] - where $K_{o}$ is the modified Bessel function of order zero. As is well known [25]

$$
K_{o}(z)=-\ln z-\frac{z^{2}}{4} \ln z-\frac{z^{4}}{64} \ln z-\ldots, \quad K_{1}(z)=\frac{1}{z}+\frac{z}{2} \ln z+\frac{z^{2}}{16} \ln z+\ldots
$$

and

$$
K_{o}(z)=\sqrt{\frac{\pi}{2 z}} e^{-z}+\ldots, \quad K_{1}(z)=\sqrt{\frac{\pi}{2 z}} e^{-z}+\ldots .
$$

are the expansions in series if $z \rightarrow 0$ and the asymptotic expansions if $z \rightarrow \infty$. In addition

$$
\begin{gathered}
\stackrel{M}{\Delta}=\stackrel{Q}{\Delta}=\frac{d^{2}}{d R^{2}}+\frac{1}{R} \frac{d}{d R}, \quad \frac{d K_{o}(k R)}{d R}=-k K_{1}(k R), \\
\frac{d K_{1}(k R)}{d R}=-k\left[K_{o}(k R)+\frac{1}{k R} K_{1}(k R)\right] .
\end{gathered}
$$

Here the $Q$ or $M$ superscript means that the differentiation applies with respect to the coordinates of the point $Q$ or $M$. We will continue to use this notation below. Let

$$
\mathcal{E}(z)=\left(K_{1}(z)-1 / z\right) / z \quad \text { and } \quad \mathcal{D}(z)=K_{o}(z)+2\left(K_{1}(z)-1 / z\right) / z
$$

be further functions that simplify notation. Substituting the expansions in series (53) we have

$$
\lim _{z \rightarrow 0} \mathcal{E}(z)=\frac{1}{2} \ln z, \quad \lim _{z \rightarrow 0} \mathcal{D}(z)=-\frac{z^{2}}{8} \ln z .
$$

Using equations (55) and (56) we obtain

$$
\frac{d \mathcal{E}(k R)}{d R}=-\frac{\mathcal{D}(k R)}{R} \quad \text { and } \quad \frac{d \mathcal{D}(k R)}{d R}=-k K_{1}(k R)-\frac{2}{R} \mathcal{D}(k R) .
$$

It is also not too difficult to check that the following equations hold:

$$
\partial_{\alpha}=\frac{r_{\alpha}}{R} \frac{d}{d R}, \quad \partial_{\alpha} \partial_{\beta}=\frac{1}{R^{2}}\left[r_{\alpha} r_{\beta} \frac{d^{2}}{d R^{2}}+\frac{\delta_{\alpha \beta} R^{2}-r_{\alpha} r_{\beta}}{R} \frac{d}{d R}\right],
$$




$$
\begin{aligned}
& \partial_{\alpha} \chi=-\frac{a}{8 \pi k^{4}}\left\{k^{2} r_{\alpha}(2 \ln R+1)-\frac{4 k r_{\alpha}}{R}\left[K_{1}(k R)-\frac{1}{k R}\right]\right\}= \\
& =-\frac{a}{8 \pi k^{4}}\left\{k^{2} r_{\alpha}(2 \ln R+1)-\frac{4 k r_{\alpha}}{R} \mathcal{E}(k R)\right\}, \\
& \partial_{\alpha} \partial_{\beta} \chi=\frac{a}{8 \pi k^{4}}\left\{k^{2}\left[2 \delta_{\alpha \beta} \ln R+\frac{\delta_{\alpha \beta} R^{2}+2 r_{\alpha} r_{\beta}}{R^{2}}\right]-\right. \\
& \left.-\frac{4 k}{R^{3}}\left[R^{2} \delta_{\alpha \beta}-2 r_{\alpha} r_{\beta}\right]\left[K_{1}(k R)-\frac{1}{k R}\right]+\frac{4 k^{2} r_{\alpha} r_{\beta}}{R^{2}} K_{0}(k R)\right\}, \\
& \Delta \chi=-\frac{a}{2 \pi k^{2}}\left[\ln R+K_{0}(k R)+1\right], \quad \partial_{\alpha} \Delta \chi=\frac{a}{2 \pi} r_{\alpha} \mathcal{E}(k R), \\
& \partial_{\alpha} \partial_{\beta} \Delta \chi=\frac{a}{2 \pi}\left[\delta_{\alpha \beta} \mathcal{E}(k R)-\frac{r_{\alpha} r_{\beta}}{R^{2}} \mathcal{D}(k R)\right], \quad \Delta \Delta \chi=-\frac{a}{2 \pi} K_{0}(k R) .
\end{aligned}
$$

Combining relations (43), (46) and (52) we have

$$
\begin{aligned}
& \mathfrak{u}_{1}=e_{1} \underbrace{D_{11} \chi}_{\mathfrak{U}_{11}}+e_{2} \underbrace{D_{12} \chi}_{\mathfrak{U}_{12}}+e_{3} \underbrace{D_{13} \chi}_{\mathfrak{U}_{12}}= \\
& =e_{1}\left\{\left[1-\mathfrak{l}^{2} \Delta+\left(\mathfrak{l}^{2}-\mathfrak{a}\right) \partial_{1}^{2}\right] \Delta-\partial_{1}^{2}\right\} \chi+e_{2}\left[\left(\mathfrak{l}^{2}-\mathfrak{a}\right) \Delta \partial_{1} \partial_{2}-\partial_{1} \partial_{2}\right] \chi+e_{3}\left\{\left(1-\mathfrak{l}^{2} \Delta\right) \partial_{2}\right\} \chi,
\end{aligned}
$$

$$
\begin{aligned}
\mathfrak{u}_{2} & =e_{1} \underbrace{D_{21} \chi}_{\mathfrak{U}_{21}}+e_{2} \underbrace{D_{22} \chi}_{\mathfrak{U}_{22}}+e_{3} \underbrace{D_{23} \chi}_{\mathfrak{U}_{23}}= \\
& =e_{1}\left[\left(\mathfrak{l}^{2}-\mathfrak{a}\right) \Delta \partial_{2} \partial_{1}-\partial_{2} \partial_{1}\right] \chi+e_{2}\left\{\left[1-\mathfrak{l}^{2} \Delta+\left(\mathfrak{l}^{2}-\mathfrak{a}\right) \partial_{1}^{2}\right] \Delta-\partial_{2}^{2}\right\} \chi-e_{3}\left(1-\mathfrak{l}^{2} \Delta\right) \partial_{1} \chi,
\end{aligned}
$$

$$
\begin{aligned}
\mathfrak{u}_{3}=e_{1} \underbrace{D_{31} \chi}_{\mathfrak{U}_{31}}+e_{2} \underbrace{D_{32} \chi}_{\mathfrak{U}_{32}}+e_{3} \underbrace{D_{33} \chi}_{\mathfrak{U}_{33}}= \\
=e_{1}\left\{-\left(1-\mathfrak{l}^{2} \Delta\right) \partial_{2}\right\} \chi+e_{2}\left(1-\mathfrak{l}^{2} \Delta\right) \partial_{1} \chi+e_{3}\left\{\left(1-\mathfrak{l}^{2} \Delta\right)(1-\mathfrak{a} \Delta)\right\} \chi .
\end{aligned}
$$

We can check using the derivatives (55) and (59) and the asymptotic relations (53) that only $\Delta \Delta \chi$ and $\Delta \partial^{2} \chi / \partial x_{\alpha} \partial x_{\beta}$ are singular among the derivatives $\Delta \Delta \chi, \Delta \partial^{2} \chi / \partial x_{\alpha} \partial x_{\beta}, \Delta \partial \chi / \partial x_{\alpha}, \Delta \chi, \partial^{2} \chi / \partial x_{\alpha} \partial x_{\beta}$ in equations $(60 \mathrm{a}, \mathrm{b}, \mathrm{c})$ if $R \rightarrow 0$ :

$$
\Delta \Delta \chi=\frac{a}{2 \pi} \ln R, \quad \Delta \partial^{2} \chi / \partial x_{\alpha} \partial x_{\beta}=\frac{a}{2 \pi} \delta_{\alpha \beta} \ln R .
$$

Omitting the formal transformations, we obtain from equations (46), (52), (55) and (56) that

$$
\mathfrak{u}_{l}=e_{k}(Q) \mathfrak{U}_{k l}(M, Q)
$$

in which

$$
\begin{gathered}
\mathfrak{U}_{\alpha \beta}(M, Q)=\frac{a}{2 \pi k^{2}}\left\{\delta_{\alpha \beta}\left[\frac{1}{2} \ln R+\frac{3}{4}+\mathfrak{a} k^{2} \mathcal{E}(k R)\right]-\frac{r_{\alpha} r_{\beta}}{R^{2}}\left[\frac{1}{2}+\mathfrak{a} k^{2} \mathcal{D}(k r)\right]\right\} \\
\mathfrak{U}_{3 \alpha}(M, Q)=-\mathfrak{U}_{\alpha 3}(M, Q)=\frac{a}{2 \pi k^{2}}(-1)^{(\alpha)} r_{3-\alpha}\left(\frac{1}{2} \ln R+\frac{1}{4}\right) \\
\mathfrak{U}_{33}(M, Q)=-\frac{a}{2 \pi k^{2}}\left\{\frac{1}{4} R^{2} \ln R-\left(\frac{1}{k^{2}}+\mathfrak{a}\right)-\mathfrak{a} \ln R\right\}
\end{gathered}
$$


are the elements of the matrix of fundamental solutions.

REMARK 10.: The fundamental solution $\mathfrak{U}_{k l}(M, Q)$ satisfies the symmetry and asymmetry conditions

$$
\mathfrak{U}_{k l}(M, Q)=\mathfrak{U}_{k l}(Q, M), \quad \mathfrak{U}_{\alpha \beta}(M, Q)=\mathfrak{U}_{\beta \alpha}(M, Q), \quad \mathfrak{U}_{3 \alpha}(M, Q)=-\mathfrak{U}_{\alpha 3}(M, Q) .
$$

REMARK 11.: The [rows] columns $\}$ of the fundamental solution $\mathfrak{U}_{k l}(M, Q)$ as three dimensional vectors satisfy the fundamental equation (40) if $[M]\{Q\}$ is the independent variable and $\{Q\}[M]$ is fixed.

REMARK 12.: Using (57), which gives the singular part of $\mathcal{E}(z)$, and the formulas providing the material constants (38) and (50) we obtain the decomposition of $\mathfrak{U}_{k l}(M, Q)$ into singular and nonsingular parts:

$$
\mathfrak{U}_{k l}(M, Q)=\stackrel{S}{\mathfrak{U}} k l(M, Q)+\stackrel{N}{\mathfrak{U}}_{k l}(M, Q)
$$

where

$$
\stackrel{S}{\mathfrak{U}_{k l}}(M, Q)=\frac{1}{2 \pi}\left[\begin{array}{lll}
b & 0 & 0 \\
0 & b & 0 \\
0 & 0 & d
\end{array}\right] \ln R \quad \text { in which } \quad b=\frac{\mu}{1-\nu}+\frac{2 \alpha \mu}{\alpha+\mu} \quad \text { and } \quad d=\gamma+\varepsilon .
$$

\section{Fundamental solutions of order two}

\subsection{Calculating stresses from the fundamental solution of order one}

In the absence of body forces the particular solutions $\stackrel{o}{t}_{\pi \rho}$ and $\stackrel{o}{\mu}_{\nu 3}$ are equal to zero in equations (6a) and (6b). Substituting the stress functions (62) into equations (6a,b), which give the force and couple stresses in terms of stress functions, we have

$$
\begin{gathered}
t_{11}=\mathfrak{u}_{1} \partial_{2}=e_{l}(Q) \underbrace{\mathfrak{U}_{l 1}(M, Q) \partial_{2}}_{\mathcal{S}_{l 1}(M, Q)}, \quad t_{12}=\mathfrak{u}_{2} \partial_{2}=e_{l}(Q) \underbrace{\mathfrak{U}_{l 2}(M, Q) \partial_{2}}_{\mathcal{S}_{l 2}(M, Q)} \\
t_{21}=-\mathfrak{u}_{1} \partial_{1}=-e_{l}(Q) \underbrace{\mathfrak{U}_{l 1}(M, Q) \partial_{1}}_{-\mathcal{S}_{l 3}(M, Q)}, \quad t_{22}=-\mathfrak{u}_{2} \partial_{1}=-e_{l}(Q) \underbrace{\mathfrak{U}_{l 2}(M, Q) \partial_{1}}_{-\mathcal{S}_{l 4}(M, Q)} \\
\mu_{13}=\mathfrak{u}_{3} \partial_{2}-\mathfrak{u}_{1}=e_{l}(Q) \underbrace{\mathfrak{U}_{l 3}(M, Q) \partial_{2}}_{\mathcal{M}_{l 1}(M, Q)}-e_{l}(Q) \mathfrak{U}_{l 1}(M, Q) \\
\mu_{23}=\mathfrak{u}_{3} \partial_{2}-\mathfrak{u}_{2}=e_{l}(Q) \underbrace{\mathfrak{U}_{l 3}(M, Q) \partial_{1}}_{\mathcal{M}_{l 2}(M, Q)}-e_{l}(Q) \mathfrak{U}_{l 1}(M, Q) .
\end{gathered}
$$

Appendix A contains the matrices $\mathcal{S}_{l N}(M, Q), N=1,2,3,4$, and $\mathcal{M}_{l \kappa}(M, Q)$, since these relations can also be used in those equations which provide the stresses at the the inner points.

Using (63a), (63b) and (63c), after further derivation we find that the force stresses are

$$
\begin{aligned}
& t_{\pi \alpha}(M, Q)=\frac{a}{2 \pi k^{2}} \epsilon_{\pi \rho 3} \frac{1}{R^{2}}\left\{\frac{1}{2}\left(\delta_{\alpha \beta} r_{\rho}-\delta_{\alpha \rho} r_{\beta}-\delta_{\beta \rho} r_{\alpha}+2 \frac{r_{\alpha} r_{\beta} r_{\rho}}{R^{2}}\right)-\right. \\
&-\mathfrak{a} k^{2}\left(\delta_{\alpha \beta} r_{\rho}+\delta_{\alpha \rho} r_{\beta}+\right.\left.\left.\delta_{\beta \rho} r_{\alpha}-4 \frac{r_{\alpha} r_{\beta} r_{\rho}}{R^{2}}\right) \mathcal{D}(k R)+\mathfrak{a} k^{2} \frac{r_{\alpha} r_{\beta} r_{\rho}}{R} k K_{1}(k R)\right\} e_{\beta}(Q)+ \\
&+\frac{a}{2 \pi k^{2}} \epsilon_{\pi \rho 3}(-1)^{(\alpha)}\left[\delta_{3-\alpha, \rho}\left(\frac{1}{2} \ln R+\frac{1}{4}\right)+\frac{r_{3-\alpha} r_{\rho}}{2 R^{2}}\right] e_{3}(Q)
\end{aligned}
$$


while the couple stresses are

$$
\begin{gathered}
\mu_{\pi 3}(M, Q)=-\frac{a}{2 \pi k^{2}}\left\{(-1)^{(\beta)} \epsilon_{\pi \rho 3}\left[\delta_{3-\beta, \rho}\left(\frac{1}{2} \ln R+\frac{1}{4}\right)+\frac{r_{3-\beta} r_{\rho}}{2 R^{2}}\right] e_{\beta}(Q)+\right. \\
+\epsilon_{\pi \rho 3}\left[r_{\rho}\left(\frac{1}{2} \ln R+\frac{1}{4}\right)-\mathfrak{a} \frac{r_{\rho}}{R^{2}}\right] e_{3}(Q)+ \\
\left.+\left\{\delta_{\pi \beta}\left[\frac{1}{2} \ln R+\frac{3}{4}+\mathfrak{a} k^{2} \mathcal{E}(k R)\right]-\frac{r_{\pi} r_{\beta}}{R^{2}}\left[\frac{1}{2}+\mathfrak{a} k^{2} \mathcal{D}(k R)\right]\right\} e_{\beta}(Q)+(-1)^{(\pi)} r_{3-\pi}\left(\frac{1}{2} \ln R+\frac{1}{4}\right) e_{3}(Q)\right\}
\end{gathered}
$$

which belong to the fundamental solutions. Note that in the above equations the Greek powers in parenthesis, i.e., $(\alpha)$ and $(\beta)$, coincide with the free index $\alpha$ and the summation index $\beta$.

\subsection{The strains that belong to the fundamental solution of order one.}

Substituting the force and couple stresses (7a) and (7b) into the HoOKE law we obtain the strain tensor $\gamma_{\kappa \lambda}$ and the curvature twist tensor $\kappa_{\nu 3}$ :

$$
\begin{aligned}
& \gamma_{11}=\mathcal{C} \mathcal{G}_{11 k}(M, Q) e_{k}(Q), \quad \mathcal{C}=a / 4 \pi \mu k^{2} R^{2}, \\
& \mathcal{G}_{111}=\frac{1}{2}\left[(1-2 \nu) r_{2}+2 \frac{r_{1}^{2} r_{2}}{R^{2}}\right]-\mathfrak{a} k^{2}\left(r_{2}-4 \frac{r_{1}^{2} r_{2}}{R^{2}}\right) \mathcal{D}(k R)+\mathfrak{a} k^{2} \frac{r_{1}^{2} r_{2}}{R} k K_{1}(k R), \\
& \mathcal{G}_{112}=-\frac{1}{2}\left[(1-2 \nu) r_{1}-2 \frac{r_{1} r_{2}^{2}}{R^{2}}\right]-\mathfrak{a} k^{2}\left(r_{1}-4 \frac{r_{1} r_{2}^{2}}{R^{2}}\right) \mathcal{D}(k R)+\mathfrak{a} k^{2} \frac{r_{1} r_{2}^{2}}{R} k K_{1}(k R), \\
& 2 \mathcal{G}_{113}=-(1-2 \nu) R^{2}(\ln R+0.5)+\nu R^{2}-r_{2}^{2}, \\
& \gamma_{12}=\mathcal{C} \mathcal{G}_{12 k}(M, Q) e_{k}(Q) / 2, \quad \gamma_{21}=\mathcal{C} \mathcal{G}_{21 k}(M, Q) e_{k}(Q) / 2, \\
& \begin{array}{l}
\mathcal{G}_{121} \\
\mathcal{G}_{211}
\end{array}=r_{1}\left[\frac{r_{2}^{2}-r_{1}^{2}}{R^{2}}+2 \mathfrak{a} k^{2}\left(1+2 \frac{r_{2}^{2}-r_{1}^{2}}{R^{2}}\right) \mathcal{D}(k R)+\mathfrak{a} k^{2} \frac{r_{2}^{2}-r_{1}^{2}}{R} k K_{1}(k R)\right] \pm \frac{\mu}{\alpha} \mathfrak{a} k^{2} r_{1} R k K_{1}(k R), \\
& \begin{array}{l}
\mathcal{G}_{122} \\
\mathcal{G}_{212}
\end{array}=r_{2}\left[\frac{r_{2}^{2}-r_{1}^{2}}{R^{2}}-2 \mathfrak{a} k^{2}\left(1-2 \frac{r_{2}^{2}-r_{1}^{2}}{R^{2}}\right) \mathcal{D}(k R)+\mathfrak{a} k^{2} \frac{r_{2}^{2}-r_{1}^{2}}{R} k K_{1}(k R)\right] \pm \frac{\mu}{\alpha} \mathfrak{a} k^{2} r_{2} R k K_{1}(k R), \\
& \mathcal{G}_{123}=\mathcal{G}_{213}=r_{1} r_{2} \\
& \gamma_{22}=\mathcal{C} \mathcal{G}_{22 k}(M, Q) e_{k}(Q), \\
& \mathcal{G}_{221}=\frac{1}{2}\left[(1-2 \nu) r_{2}-2 \frac{r_{1}^{2} r_{2}}{R^{2}}\right]+\mathfrak{a} k^{2}\left(r_{2}-4 \frac{r_{1}^{2} r_{2}}{R^{2}}\right) \mathcal{D}(k R)-\mathfrak{a} k^{2} \frac{r_{1}^{2} r_{2}}{R} k K_{1}(k R), \\
& \mathcal{G}_{222}=-\frac{1}{2}\left[(1-2 \nu) r_{1}+2 \frac{r_{1} r_{2}^{2}}{R^{2}}\right]+\mathfrak{a} k^{2}\left(r_{1}-4 \frac{r_{1} r_{2}^{2}}{R^{2}}\right) \mathcal{D}(k R)-\mathfrak{a} k^{2} \frac{r_{1} r_{2}^{2}}{R} k K_{1}(k R), \\
& 2 \mathcal{G}_{223}=-(1-2 \nu) R^{2}(\ln R+0.5)+\nu R^{2}-r_{1}^{2}, \\
& \kappa_{13}=\frac{a}{2 \pi k^{2}} \frac{1}{\gamma+\varepsilon} \mathcal{K}_{13 k}(M, Q) e_{k}(Q), \\
& \mathcal{K}_{131}=-\mathfrak{a} k^{2}\left[\mathcal{E}(k r)-\frac{r_{1}^{2}}{R^{2}} \mathcal{D}(k R)\right], \quad \mathcal{K}_{132}=\frac{r_{1} r_{2}}{R^{2}} \mathfrak{a} k^{2} \mathcal{D}(k R), \quad \mathcal{K}_{133}=\mathfrak{a} \frac{r_{2}}{R^{2}} \\
& \kappa_{23}=\frac{a}{2 \pi k^{2}} \frac{1}{\gamma+\varepsilon} \mathcal{K}_{23 k}(M, Q) e_{k}(Q), \\
& \mathcal{K}_{231}=\frac{r_{1} r_{2}}{R^{2}} \mathfrak{a} k^{2} \mathcal{D}(k R), \quad \mathcal{K}_{232}=-\mathfrak{a} k^{2}\left[\mathcal{E}(k r)-\frac{r_{2}^{2}}{R^{2}} \mathcal{D}(k R)\right], \quad \mathcal{K}_{233}=-\mathfrak{a} \frac{r_{1}}{R^{2}}
\end{aligned}
$$


The dual stresses are defined by equations

$$
\mathfrak{t}_{\lambda}=\tau_{1} \gamma_{1 \lambda}+\tau_{2} \gamma_{2 \lambda} \quad \text { and } \quad \mathfrak{t}_{3}=\tau_{1} \kappa_{13}+\tau_{2} \kappa_{23} .
$$

Note that these quantities constitute the right sides of the strain boundary conditions (13a) and (13b). We now introduce the following additional notation

$$
\begin{gathered}
\Phi_{\rho 1}=\frac{1}{2}\left[(1-2 \nu)-(-1)^{(\rho)} 2 \frac{r_{\rho}^{2}}{R^{2}}\right]+(-1)^{(\rho)} \mathfrak{a} k^{2}\left[\left(1-4 \frac{r_{\rho}^{2}}{R^{2}}\right) \mathcal{D}(k R)-\frac{r_{\rho}^{2}}{R} k K_{1}(k R)\right] \\
\Phi_{\rho 2}=\frac{1}{2}\left[(1-2 \nu)+(-1)^{(\rho)} 2 \frac{r_{\rho}^{2}}{R^{2}}\right]-(-1)^{(\rho)} \mathfrak{a} k^{2}\left[\left(1-4 \frac{r_{\rho}^{2}}{R^{2}}\right) \mathcal{D}(k R)-\frac{r_{\rho}^{2}}{R} k K_{1}(k R)\right] \\
\Phi_{3 \kappa}=(1-2 \nu) R^{2}\left(\frac{1}{2} \ln R+\frac{1}{4}\right)-\nu \frac{R^{2}}{2}+\frac{r_{3-\kappa}^{2}}{2} \\
\Psi_{\rho \kappa}=\frac{1}{2}\left\{\frac{r_{2}^{2}-r_{1}^{2}}{R^{2}}-(-1)^{(\rho)} 2 \mathfrak{a} k^{2}\left(1-(-1)^{(\rho)} 2 \frac{r_{2}^{2}-r_{1}^{2}}{R^{2}}\right) \mathcal{D}(k R)+\mathfrak{a} k^{2} \frac{r_{2}^{2}-r_{1}^{2}}{R} k K_{1}(k R)\right\}+ \\
+(-1)^{(\kappa)} \frac{\mu}{2 \alpha} \mathfrak{a} k^{2} R k K_{1}(k R) \\
\mathcal{K}_{\rho 3}=-\mathfrak{a} k^{2}\left[R^{2} \mathcal{E}(k R)-r_{\rho}^{2} \mathcal{D}(k R)\right] \\
\Psi_{13}=\Psi_{23}=r_{1} r_{2} \mathfrak{a} k^{2} \mathcal{D}(k R)
\end{gathered}
$$

Substitute now the strains (70a),...,(74b) into the definitions of the dual stresses (75). If we then use the notation $(76), \ldots,(81)$, we obtain

$$
\mathfrak{t}_{k}=e_{l}(Q) \mathfrak{T}_{l k}\left(M_{\circ}, Q\right),
$$

where

$$
\begin{gathered}
\mathfrak{T}_{l k}\left(M_{\circ}, Q\right)=\frac{1}{2 \pi(1-\nu) R^{2}} \times \\
\times\left[\begin{array}{ccc}
-n_{2} r_{2} \Phi_{11}+n_{1} r_{1} \Psi_{11} & n_{1} r_{2} \Phi_{12}-n_{2} r_{1} \Psi_{12} & \frac{2 \mu}{(\gamma+\varepsilon)}\left(-n_{2} \mathcal{K}_{13}+n_{1} \Psi_{13}\right) \\
n_{2} r_{1} \Phi_{21}+n_{1} r_{2} \Psi_{21} & -n_{1} r_{1} \Phi_{22}-n_{2} r_{2} \Psi_{22} & -\frac{2 \mu}{(\gamma+\varepsilon)}\left(-n_{1} \mathcal{K}_{23}+n_{2} \Psi_{23}\right) \\
n_{2} \Phi_{31}+\frac{1}{2} n_{1} r_{1} r_{2} & -n_{1} \Phi_{32}-\frac{1}{2} n_{2} r_{1} r_{2} & -\frac{2 \mu}{(\gamma+\varepsilon)} \mathfrak{a}\left(n_{1} r_{1}+n_{2} r_{2}\right)
\end{array}\right] .
\end{gathered}
$$

The last equation is the formula for calculating the dual stresses $\mathfrak{t}_{k}$ (brought into existence by the incompatibility $\left.e_{l}=e_{l}(Q)\right)$ at the point $M_{o}$ on the boundary where the outward unit normal is $n_{l}=n_{l}\left(M_{o}\right)$.

REMARK 13.: The matrix $\mathfrak{T}_{l k}\left(M_{\circ}, Q\right)$ is that of the fundamental solutions of order two.

REMARK 14.: Matrix $\mathfrak{T}_{l k}\left(M_{\circ}, Q\right)$ can also be resolved into a strongly singular and a non-strongly singular part:

$$
\mathfrak{T}_{l k}\left(M_{\circ}, Q\right)=\stackrel{S}{\mathfrak{T}_{l k}}\left(M_{\circ}, Q\right)+\stackrel{N}{\mathfrak{T}_{l k}}\left(M_{\circ}, Q\right),
$$

where

$$
\underset{\mathfrak{T}_{l k}}{S}\left(M_{\circ}, Q\right)=-\frac{1}{2 \pi}\left[\begin{array}{ccl}
\frac{\partial \ln R}{\partial n_{o}} & g \frac{\partial \ln R}{\partial s_{o}} & 0 \\
-g \frac{\partial \ln R}{\partial s_{o}} & \frac{\partial \ln R}{\partial n_{o}} & 0 \\
0 & 0 & \frac{\partial \ln R}{\partial n_{o}}
\end{array}\right]
$$


and

$$
g=\frac{1}{2(1-\nu)}-\frac{2 \mu}{\alpha+\mu}
$$

The singularity of matrix $\stackrel{N}{\mathfrak{T}}_{k l}(M, Q)$ is weaker than that of matrix $\stackrel{S}{\mathfrak{T}}_{k l}(\stackrel{o}{M}, Q)$.

This statement can be proved as follows. Recall that the dual stresses $\mathfrak{t}_{k}$ are obtained from equations (35) provided that the stress functions $\mathcal{F}_{1}, \mathcal{F}_{2}$ and $\mathcal{H}$ are those obtained from the fundamental solutions. Consequently we can write

$$
\begin{aligned}
\mathfrak{t}_{1} & =e_{1} \underbrace{\left\{\frac{\tau_{1}}{2 \mu}\left[(1-\nu)\left(D_{11} \partial_{2} \chi\right)+\nu\left(D_{21} \partial_{1} \chi\right)\right]+\tau_{2}\left[\left(\frac{1}{4 \mu}-\frac{1}{4 \alpha}\right)\left(D_{21} \partial_{2} \chi\right)-\left(\frac{1}{4 \mu}+\frac{1}{4 \alpha}\right)\left(D_{11} \partial_{1} \chi\right)\right]\right\}}_{\mathfrak{T}_{11}}+ \\
+ & e_{2} \underbrace{\left\{\frac{\tau_{1}}{2 \mu}\left[(1-\nu)\left(D_{12} \partial_{2} \chi\right)+\nu\left(D_{22} \partial_{1} \chi\right)\right]+\tau_{2}\left[\left(\frac{1}{4 \mu}-\frac{1}{4 \alpha}\right)\left(D_{22} \partial_{2} \chi\right)-\left(\frac{1}{4 \mu}+\frac{1}{4 \alpha}\right)\left(D_{12} \partial_{1} \chi\right)\right]\right\}}_{\mathfrak{T}_{21}}+ \\
& +e_{3} \underbrace{\left\{\frac{\tau_{1}}{2 \mu}\left[(1-\nu)\left(D_{13} \partial_{2} \chi\right)+\nu\left(D_{23} \partial_{1} \chi\right)\right]+\tau_{2}\left[\left(\frac{1}{4 \mu}-\frac{1}{4 \alpha}\right)\left(D_{23} \partial_{2} \chi\right)-\left(\frac{1}{4 \mu}+\frac{1}{4 \alpha}\right)\left(D_{13} \partial_{1} \chi\right)\right]\right\}}_{\mathfrak{T}_{31}}
\end{aligned}
$$

$$
\begin{aligned}
& \mathfrak{t}_{2}=e_{1} \underbrace{\left\{-\frac{\tau_{2}}{2 \mu}\left[(1-\nu)\left(D_{21} \partial_{1} \chi\right)+\nu\left(D_{11} \partial_{2} \chi\right)\right]+\tau_{1}\left[\left(\frac{1}{4 \mu}-\frac{1}{4 \alpha}\right)\left(D_{21} \partial_{2} \chi\right)-\left(\frac{1}{4 \mu}+\frac{1}{4 \alpha}\right)\left(D_{11} \partial_{1} \chi\right)\right]\right\}}_{\mathfrak{T}_{12}}+ \\
& +e_{2} \underbrace{\left\{-\frac{\tau_{2}}{2 \mu}\left[(1-\nu)\left(D_{22} \partial_{1} \chi\right)+\nu\left(D_{12} \partial_{2} \chi\right)\right]+\tau_{1}\left[\left(\frac{1}{4 \mu}-\frac{1}{4 \alpha}\right)\left(D_{22} \partial_{2} \chi\right)-\left(\frac{1}{4 \mu}+\frac{1}{4 \alpha}\right)\left(D_{12} \partial_{1} \chi\right)\right]\right\}}_{\mathfrak{T}_{22}}+ \\
& +e_{3} \underbrace{\left\{-\frac{\tau_{2}}{2 \mu}\left[(1-\nu)\left(D_{23} \partial_{1} \chi\right)+\nu\left(D_{13} \partial_{2} \chi\right)\right]+\tau_{1}\left[\left(\frac{1}{4 \mu}-\frac{1}{4 \alpha}\right)\left(D_{23} \partial_{2} \chi\right)-\left(\frac{1}{4 \mu}+\frac{1}{4 \alpha}\right)\left(D_{13} \partial_{1} \chi\right)\right]\right\}}_{\mathfrak{T}_{32}}
\end{aligned}
$$

$$
\begin{array}{r}
\mathfrak{t}_{3}=e_{1} \underbrace{\frac{1}{\gamma+\varepsilon}\left\{\tau_{1}\left[\left(D_{31} \partial_{2} \chi\right)-\left(D_{11} \chi\right)\right]+\tau_{2}\left[\left(-D_{31} \partial_{1} \chi\right)-\left(D_{21} \chi\right)\right]\right\}}_{\mathfrak{T}_{13}}+ \\
+e_{2} \underbrace{\gamma}_{\mathfrak{T}_{23} \frac{1}{\gamma+\varepsilon}\left\{\tau_{1}\left[\left(D_{32} \partial_{2} \chi\right)-\left(D_{12} \chi\right)\right]+\tau_{2}\left[\left(-D_{32} \partial_{1} \chi\right)-\left(D_{22} \chi\right)\right]\right\}}+ \\
+e_{3} \underbrace{\frac{1}{\gamma+\varepsilon}\left\{\tau_{1}\left[\left(D_{33} \partial_{2} \chi\right)-\left(D_{13} \chi\right)\right]+\tau_{2}\left[\left(-D_{33} \partial_{1} \chi\right)-\left(D_{23} \chi\right)\right]\right\}}_{\mathfrak{T}_{33}}
\end{array}
$$

Note that these relations provide the elements of the fundamental solutions of order two directly in terms the GALJORKIN function $\chi$.

In the sequel we shall demonstrate how to determine the singular parts for the diagonal element $\mathfrak{T}_{11}$ and the off-diagonal element $\mathfrak{T}_{12}$. First we substitute the derivatives of $\chi$ from the equations (59). Then we keep only those terms on the basis of equation (61) which contain the derivatives of the weakly singular $\ln R$. We remark that the interchangeability of the derivations might be needed to identify the 
nonsingular terms. Finally we use definitions (10), (38) and (50) of the material constants. These steps imply

$$
\begin{aligned}
& \stackrel{\mathfrak{T}}{11}_{11}^{S}=\frac{\tau_{1}}{2 \mu}\left[(1-\nu)\left(D_{11} \partial_{2} \chi\right)+\nu\left(D_{21} \partial_{1} \chi\right)\right]+\tau_{2}\left[\left(\frac{1}{4 \mu}-\frac{1}{4 \alpha}\right)\left(D_{21} \partial_{2} \chi\right)-\left(\frac{1}{4 \mu}+\frac{1}{4 \alpha}\right)\left(D_{11} \partial_{1} \chi\right)\right]= \\
&=\frac{\tau_{1}}{2 \mu}\{(1-\nu)\left[-\left(1-\mathfrak{l}^{2} \Delta+\left(\mathfrak{l}^{2}-\mathfrak{a}\right) \partial_{1}^{2}\right) \Delta+\partial_{1}^{2}\right] \partial_{2} \chi+\underbrace{\nu\left[\left(-\left(\mathfrak{l}^{2}-\mathfrak{a}\right) \Delta \partial_{2} \partial_{1}+\partial_{2} \partial_{1}\right) \partial_{1} \chi\right]}_{\text {non singular }}\}+ \\
&+\tau_{2}[\underbrace{\left.\left(\frac{1}{4 \mu}-\frac{1}{4 \alpha}\right)\left[\left(-\left(\mathfrak{l}^{2}-\mathfrak{a}\right) \Delta \partial_{2} \partial_{1}+\partial_{2} \partial_{1}\right) \partial_{2}\right] \chi\right)}_{\text {non singular }}-\left(\frac{1}{4 \mu}+\frac{1}{4 \alpha}\right)\left[-\left(1-\mathfrak{l}^{2} \Delta+\left(\mathfrak{l}^{2}-\mathfrak{a}\right) \partial_{1}^{2}\right) \Delta+\partial_{1}^{2}\right] \partial_{1} \chi] \simeq \\
& \simeq \frac{\tau_{1}}{2 \mu}\left\{(1-\nu) \mathfrak{l}^{2} \Delta \Delta \partial_{2} \chi\right\}-\tau_{2}\left[\left(\frac{1}{4 \mu}+\frac{1}{4 \alpha}\right)\left[-\left(1-\mathfrak{l}^{2} \partial_{1}^{2}-\mathfrak{l}^{2} \partial_{2}^{2}+\left(\mathfrak{l}^{2}-\mathfrak{a}\right) \partial_{1}^{2}\right) \Delta+\partial_{1}^{2}\right] \partial_{1} \chi\right] \simeq \\
& \simeq-\frac{n_{2}}{2 \mu}(1-\nu) \mathfrak{l}^{2} \Delta \Delta \partial_{2} \chi-n_{1}\left(\frac{1}{4 \mu}+\frac{1}{4 \alpha}\right) \mathfrak{a} \Delta\left(\partial_{1}^{2}+\partial_{2}^{2}\right) \partial_{1} \chi= \\
&=-n_{2}^{\frac{(1-\nu) a \mathfrak{l}^{2}}{2 \mu}} \frac{1}{2 \pi} \partial_{2} \ln R-n_{1}^{\frac{1}{4 \mu} \frac{\alpha+\mu}{\alpha \mu} \mathfrak{a} a \frac{1}{2 \pi} \partial_{2} \ln R=-\frac{1}{2 \pi} \frac{\partial \ln R}{\partial n_{o}} .} .
\end{aligned}
$$

For $\mathfrak{T}_{12}$ a similar argument yields

$$
\begin{gathered}
\stackrel{\mathfrak{T}}{S}_{12}^{S}=-\frac{\tau_{2}}{2 \mu}\left[(1-\nu)\left(D_{21} \partial_{1} \chi\right)+\nu\left(D_{11} \partial_{2} \chi\right)\right]+\tau_{1}\left[\left(\frac{1}{4 \mu}-\frac{1}{4 \alpha}\right)\left(D_{21} \partial_{2} \chi\right)-\left(\frac{1}{4 \mu}+\frac{1}{4 \alpha}\right)\left(D_{11} \partial_{1} \chi\right)\right]= \\
=-\frac{\tau_{2}}{2 \mu}\left\{(1-\nu)\left(\left(\mathfrak{l}^{2}-\mathfrak{a}\right) \Delta \partial_{1} \partial_{2}-\partial_{1} \partial_{2}\right) \partial_{1} \chi+\nu\left[\left(1-\mathfrak{l}^{2} \Delta+\left(\mathfrak{l}^{2}-\mathfrak{a}\right) \partial_{2}^{2}\right) \Delta-\partial_{1}^{2}\right] \partial_{2} \chi\right\}+ \\
+\tau_{1}\left[-\left(\frac{1}{4 \mu}-\frac{1}{4 \alpha}\right)\left[\left(1-\mathfrak{l}^{2} \Delta+\left(\mathfrak{l}^{2}-\mathfrak{a}\right) \partial_{1}^{2}\right) \Delta-\partial_{1}^{2}\right] \partial_{1} \chi+\left(\frac{1}{4 \mu}+\frac{1}{4 \alpha}\right)\left(\left(\mathfrak{l}^{2}-\mathfrak{a}\right) \Delta \partial_{1} \partial_{2}-\partial_{1} \partial_{2}\right) \partial_{2} \chi\right] \simeq \\
\simeq \frac{\tau_{2}}{4 \mu \alpha}\left\{2 \alpha \nu \mathfrak{l}^{2} \partial_{2}^{2}\right\} \partial_{2} \chi+\frac{\tau_{1}}{4 \mu \alpha}\left[(\alpha-\mu)\left(\mathfrak{l}^{2} \partial_{2}^{2}+\mathfrak{a} \partial_{1}^{2}\right) \Delta+(\alpha+\mu)\left(\mathfrak{l}^{2}-\mathfrak{a}\right) \Delta \partial_{2}^{2}\right] \partial_{1} \chi \simeq \\
\simeq \frac{\tau_{2}}{4 \mu \alpha}\left\{2 \alpha \nu \mathfrak{l}^{2} \Delta \partial_{2}^{2}-2 \mu \mathfrak{a} \Delta \partial_{1}^{2}\right\} \partial_{2} \chi+\frac{\tau_{1}}{4 \mu \alpha}\left[(\alpha-\mu)\left(\mathfrak{l}^{2} \Delta \partial_{2}^{2}+\mathfrak{a} \Delta \partial_{1}^{2}\right)+(\alpha+\mu)\left(\mathfrak{l}^{2}-\mathfrak{a}\right) \Delta \partial_{2}^{2}\right] \partial_{1} \chi= \\
=\frac{1}{4 \pi} \frac{a}{4 \mu \alpha}\left\{2 \alpha \nu \mathfrak{l}^{2} \partial_{2}^{2}-2 \mu \mathfrak{a} \partial_{1}^{2} \Delta\right\} \tau_{2} \partial_{2} \ln R+\frac{1}{4 \pi} \frac{a}{4 \mu \alpha}\left[(\alpha-\mu)\left(\mathfrak{l}^{2}+\mathfrak{a}\right)+(\alpha+\mu)\left(\mathfrak{l}^{2}-\mathfrak{a}\right)\right] \tau_{1} \partial_{1} \ln R= \\
=\frac{1}{2 \pi}\left[\frac{1}{2(1-\nu)}-\frac{2 \mu}{\alpha+\mu}\right]\left(\tau_{2} \partial_{2}+\tau_{1} \partial_{1}\right) \ln R=\frac{1}{2 \pi}\left[\frac{1}{2(1-\nu)}-\frac{2 \mu}{\alpha+\mu}\right] \frac{\partial \ln R}{\partial s_{o}} .
\end{gathered}
$$

For the other cases the procedure is basically the same.

\section{Dual Somiglian formulae for inner regions}

\subsection{The dual Somigliana identity}

In this section we assume that the region under consideration is the simple connected inner region $A^{+}$ depicted in Figure 2.

The functions $\mathcal{F}_{\psi}, \mathcal{H}, t_{\kappa \lambda}, \mu_{\nu 3}, \gamma_{\kappa \lambda}$ and $\kappa_{\nu 3}$ are called an elastic state of the region $A^{+}$if they satisfy the field equations $(6 a), \ldots,(8)$. Let

$$
\mathcal{F}_{\psi}, \mathcal{H}, t_{\kappa \lambda}, \mu_{\nu 3}, \gamma_{\kappa \lambda}, \kappa_{\nu 3}
$$

and

$$
\stackrel{*}{\mathcal{F}}_{\psi}, \stackrel{*}{\mathcal{H}}, \stackrel{*}{t}_{\kappa \lambda}, \stackrel{*}{\mu_{\nu 3}}, \stackrel{*}{\gamma}_{\kappa \lambda}, \stackrel{*}{\kappa}
$$




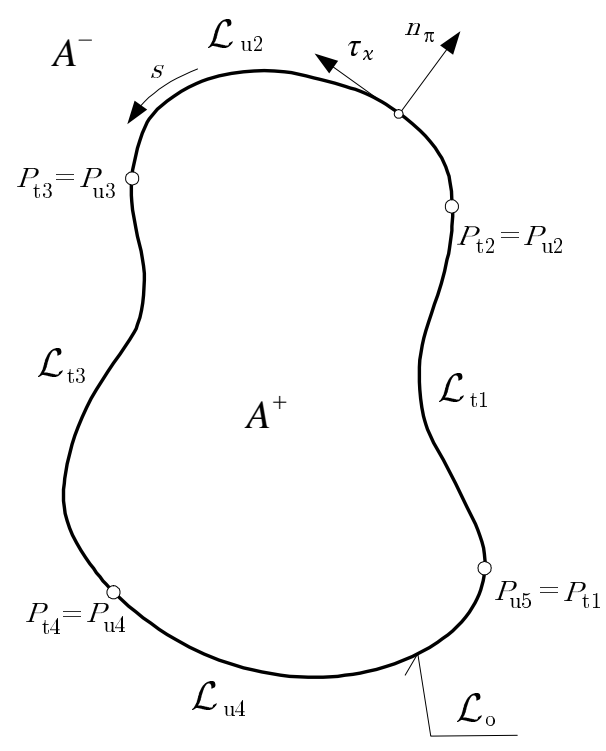

Figure 2: A simply connected inner region

be two elastic states of the region $A^{+}$. Integrating by parts the compatibility differential equations (8) (the weights are $\mathcal{F}_{\rho}$ and $\mathcal{H}^{*}$ ), using equations (6a) and (6b) and noting that because there are no body forces the particular solutions for the stresses and couple stresses are equal to zero, we obtain

$$
\begin{aligned}
\int_{A^{+}} \epsilon_{3 \mu \pi}\left(\gamma_{\pi \rho} \partial_{\mu}+\epsilon_{\pi \rho 3} \kappa_{\mu 3}\right) & \stackrel{*}{\mathcal{F}} \rho A+\int_{A^{+}} \epsilon_{3 \psi \nu} \kappa_{\nu 3} \partial_{\psi} \stackrel{*}{\mathcal{H}} d A= \\
& =\oint_{\mathcal{L}_{o}} \tau_{\pi} \gamma_{\pi \rho} \stackrel{*}{\mathcal{F}}_{\rho} d s+\oint_{\mathcal{L}_{o}} \tau_{\nu} \kappa_{\nu 3} \stackrel{*}{\mathcal{H}} d s+ \\
& +\int_{A^{+}} \epsilon_{\pi \mu 3}\left(\stackrel{*}{\mathcal{F}}_{\rho} \partial_{\mu}\right) \gamma_{\pi \rho} d A+\int_{A^{+}} \epsilon_{\nu \psi 3}\left(\stackrel{*}{\mathcal{H}} \partial_{\psi}+\epsilon_{\psi \rho 3} \stackrel{*}{\mathcal{F}}_{\rho}\right) \kappa_{\nu 3} d A
\end{aligned}
$$

REMARK 15.: The value of the area integrals on the right side does not depend on whether the asterisk is over the first or the second variable in the products. This can be seen if we express the strains $\gamma_{\pi \rho}$ and $\kappa_{\nu 3}$ in terms of stress functions using equations (6a) and (6b) which provide the stresses in terms of stress functions and then the HoOKE law.

The dual SomiglianA identity is obtained if we move the asterisks from their original position to the variables which belong to the first elastic state, and then subtract the original equation (86):

$$
\begin{aligned}
& \int_{A^{+}} \underbrace{\mathcal{F}_{\rho} \epsilon_{3 \mu \pi}\left(\stackrel{*}{\gamma}_{\pi \rho} \partial_{\mu}+\epsilon_{\pi \rho 3} \stackrel{*}{\kappa}_{\mu 3}\right)}_{\mathfrak{u}_{\rho}\left(\mathfrak{\mathcal { D }}_{\rho l} \mathfrak{u}_{l}\right)} d A-\int_{A^{+}} \underbrace{\mathcal{H} \epsilon_{3 \psi \nu}\left(\kappa_{\nu 3}^{*} \partial_{\psi}\right)}_{\mathfrak{u}_{3}\left(\mathfrak{D}_{3 l} \mathfrak{u}_{l}\right)} d A- \\
& -\int_{A^{+}} \epsilon_{3 \mu \pi}\left(\gamma_{\pi \rho} \partial_{\mu}+\epsilon_{\pi \rho 3} \kappa_{\mu 3}\right) \stackrel{*}{\mathcal{F}}{ }_{\rho} d A+\int_{A^{+}} \epsilon_{3 \psi \nu}\left(\kappa_{\nu 3} \partial_{\psi}\right) \stackrel{*}{\mathcal{H}} d A= \\
& =\oint_{\mathcal{L}_{o}} \underbrace{\mathcal{F}_{\rho} \tau_{\pi} \gamma_{\pi \rho}^{*}}_{\substack{* * \\
\mathfrak{u}_{\rho} \mathfrak{t}_{\rho}}} d s-\oint_{\mathcal{L}_{o}} \underbrace{\mathcal{H} \tau_{\nu} \kappa_{\nu 3}^{*}}_{\substack{* * \\
\mathfrak{u}_{3} \mathfrak{t}_{3}}} d s-\oint_{\mathcal{L}_{o}} \tau_{\pi} \gamma_{\pi \rho} \stackrel{*}{\mathcal{F}}_{\rho} d s+\oint_{\mathcal{L}_{o}} \tau_{\nu} \kappa_{\nu 3} \stackrel{*}{\mathcal{H}} d s .
\end{aligned}
$$

On the right hand side we have the weighted integrals of the basic equations with the dual displacements as weights. The left hand side involves the products of the quantities on which boundary conditions 
can be imposed. Consequently the SomiglianA identity can be cast into a form similar to the Green identity of the potential theory. Introduce first the notation

$$
{ }^{\prime} \mathfrak{D}_{k l}=\mathfrak{D}_{k l} /(\gamma+\epsilon) .
$$

where ${ }^{\prime} \mathfrak{D}_{k l}$ is the operator of the basic equations (34). Using (41) and (42) with equation (87), after some manipulations we obtain

$$
\int_{A^{+}}\left[\mathfrak{u}_{k}\left(' \mathfrak{D}_{k l} \mathfrak{u}_{l}\right)-\stackrel{\mathfrak{u}}{k}_{k}\left(' \mathfrak{D}_{k l} \mathfrak{u}_{l}\right)\right] d A=\oint_{\mathcal{L}_{o}}\left[\mathfrak{u}_{l}^{*} \mathfrak{t}_{l}-\mathfrak{t}_{l} \mathfrak{u}_{l}\right] d s .
$$

which has the same structure as the Green identity [26].

REMARK 16.: In the argument leading to (89) we did not use that the quantities $\stackrel{*}{\mathfrak{u}}_{k}$ and $\mathfrak{u}_{k}$ are elastic states of the region $A^{+}$. Consequently (89) always holds provided that $\mathfrak{\mathfrak { u }}_{k}$ and $\mathfrak{u}_{k}$ are arbitrary functions that can be differentiated as many times as required.

\subsection{The dual Somigliana formulae for inner regions}

To derive the dual Somigliana formulae we shall assume that $\mathfrak{u}_{l}(M)$ is an elastic state of the region $A^{+}$. Suppose that the other elastic state, denoted by $*$, is the one which belongs to the fundamental solutions:

$$
\stackrel{*}{\mathfrak{U}}_{l}(M)=e_{k}(Q) \mathfrak{U}_{k l}(M, Q), \quad \stackrel{*}{\mathfrak{t}}_{l}(M)=e_{k}(Q) \mathfrak{T}_{k l}(M, Q)
$$

The latter is singular at the point $Q$. Depending on the position of the point $Q$ relative to the region $A^{+}$, we distinguish three cases - two of them are shown in Figure 3.

1. If $Q \in A^{+}$, then we first remove the neighborhood of $Q$ with radius $R_{\varepsilon}$, denoted $A_{\varepsilon}$ and assumed to lie wholly in $A^{+}$, from $A^{+}$; and then we apply the dual Somigliana identity to the double connected domain $A^{\prime}=A^{+} \backslash A_{\varepsilon}$. Note that the contour $\mathcal{L}_{\varepsilon}$ of $A_{\varepsilon}$ and the $\operatorname{arc} \mathcal{L}_{\varepsilon}^{\prime}$, which is assumed to be the part of the contour $\mathcal{L}_{\varepsilon}$ lying within $A^{+}$coincide with each other.

2. If $Q=\stackrel{o}{Q} \in \partial A=\mathcal{L}_{o}$, then the part $A^{+} \cap A_{\varepsilon}$ of the neighborhood $A_{\varepsilon}$ of $Q$ is removed from $A^{+}$and we then apply the dual SOMIGLIANA identity to the simply connected region $A^{\prime}=A^{+} \backslash\left(A^{+} \cap A_{\varepsilon}\right)$. In this case, the contour of the simply connected region just obtained consists of two arcs, the arc $\mathcal{L}_{o}^{\prime}$ left from $\mathcal{L}_{o}$ after the removal of $A_{\varepsilon}$ and the $\operatorname{arc} \mathcal{L}_{\varepsilon}^{\prime}$, i.e., the part of $\mathcal{L}_{\varepsilon}$ that lies within $A_{i}$.

3. If $Q \notin\left(A^{+} \cup \mathcal{L}_{o}\right)$ we apply the dual Somigliana identity to the original region $A^{+}$.

Since both $\mathfrak{u}_{k}$ and $\mathfrak{u}_{k}$ are elastic states the surface integrals in (89) are identically equal to zero.
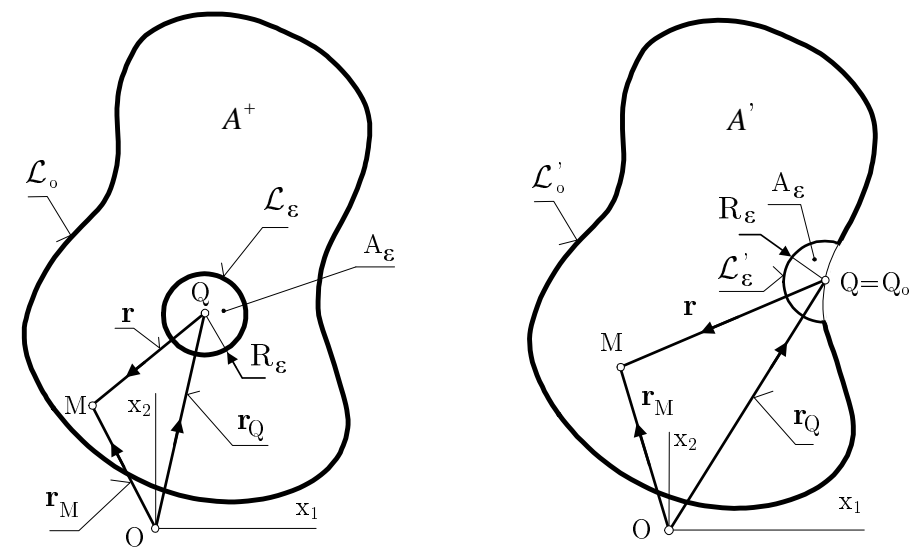

Figure 3: 
We now consider each of these three cases, focusing on the main steps of the argument.

1. If $Q \in A_{i}$ then substitute (90) into equation (89). This yields

$$
\begin{aligned}
\oint_{\mathcal{L}_{o}}\left[\mathfrak{T}_{k l}\left(M_{\circ}, Q\right) \mathfrak{u}_{l}\left(M_{\circ}\right)\right. & \left.-\mathfrak{U}_{k l}\left(M_{\circ}, Q\right) \mathfrak{t}_{l}\left(M_{\circ}\right)\right] d s_{M_{\circ}}+ \\
& +\oint_{\mathcal{L}_{\varepsilon}}\left[\mathfrak{T}_{k l}\left(M_{\circ}, Q\right) \mathfrak{u}_{l}\left(M_{\circ}\right)-\mathfrak{U}_{k l}\left(M_{\circ}, Q\right) \mathfrak{t}_{l}\left(M_{\circ}\right)\right] d s_{M_{\circ}}=0 .
\end{aligned}
$$

Substituting the singular and non-singular parts of the fundamental solutions $\mathfrak{U}_{k l}\left(M_{\circ}, Q\right)$ and $\mathfrak{T}_{k l}\left(M_{\circ}, Q\right)$ into the left sides of (92a) and (92b), using equations $(66),(84 \mathrm{a}, \mathrm{b})$ and noting that the limit of integrals involving the non-singular parts is zero, we obtain

$$
\begin{gathered}
\lim _{R_{\varepsilon} \rightarrow 0} \oint_{\mathcal{L}_{\varepsilon}} \mathfrak{T}_{k l}\left(M_{\circ}, Q\right)\left[\mathfrak{u}_{l}\left(M_{\circ}\right)-\mathfrak{u}_{l}(Q)\right] d s_{M_{\circ}}=0 \\
\lim _{R_{\varepsilon} \rightarrow 0} \oint_{\mathcal{L}_{\varepsilon}} \mathfrak{T}_{k l}\left(M_{\circ}, Q\right) d s_{M_{\circ}}=\delta_{k l} \quad \text { and } \quad \lim _{R_{\varepsilon} \rightarrow 0} \oint_{\mathcal{L}_{\varepsilon}} \mathfrak{U}_{k l}\left(M_{\circ}, Q\right) \mathfrak{t}_{l}\left(M_{\circ}\right) d s_{M_{\circ}}=0 .
\end{gathered}
$$

Substituting these limits into equation (91) yields the first dual SOMIGLIANA formula

$$
\mathfrak{u}_{k}(Q)=\oint_{\mathcal{L}_{o}} \mathfrak{U}_{k l}\left(M_{\circ}, Q\right) \mathfrak{t}_{l}\left(M_{\circ}\right) d s_{M_{\circ}}-\oint_{\mathcal{L}_{o}} \mathfrak{T}_{k l}\left(M_{\circ}, Q\right) \mathfrak{u}_{l}\left(M_{\circ}\right) d s_{M_{\circ}} .
$$

REMARK 17.: Given the dual displacements (stress functions) $\mathfrak{u}_{\lambda}\left(M_{\circ}\right)$ and dual stresses (the corresponding displacement derivatives) $\mathfrak{t}_{\lambda}\left(M_{\circ}\right)$ on the contour $\mathcal{L}_{o}$, the first dual SomigLiAnA formula provides the elastic state $u_{k}(Q)$ in terms of two determinate integrals.

2. If $Q=Q_{\circ} \in \partial A=\mathcal{L}_{o}$ then repeating the logic leading from (89) to (91) we can write

$$
\begin{aligned}
& \oint_{\mathcal{L}_{o}^{\prime}}\left[\mathfrak{T}_{k l}\left(M_{\circ}, Q_{\circ}\right) \mathfrak{u}_{l}\left(M_{\circ}\right)-\mathfrak{U}_{k l}\left(M_{\circ}, Q_{\circ}\right) \mathfrak{t}_{l}\left(M_{\circ}\right)\right] d s_{M_{\circ}}+ \\
+ & \oint_{\mathcal{L}_{\varepsilon}^{\prime}}\left[\mathfrak{T}_{k l}\left(M_{\circ}, Q_{\circ}\right) \mathfrak{u}_{l}\left(M_{\circ}\right)-\mathfrak{U}_{k l}\left(M_{\circ}, Q_{\circ}\right) \mathfrak{t}_{l}\left(M_{\circ}\right)\right] d s_{M_{\circ}}=0,
\end{aligned}
$$

where

$$
\lim _{R_{\varepsilon} \rightarrow 0} \int_{\mathcal{L}_{\varepsilon}^{\prime}} \mathfrak{T}_{k l}\left(M_{\circ}, Q_{\circ}\right) d s_{M}=c_{k l}\left(Q_{\circ}\right) .
$$

We remark that $c_{k l}\left(Q_{\circ}\right)=\delta_{k l} / 2$ if the contour $\mathcal{L}_{o}$ is smooth at at the point $Q_{\circ}$. If not then $c_{k l}\left(Q_{\circ}\right)$ depends on the angle formed by the tangents to the contour at $Q_{\circ}$. In addition

$$
\begin{gathered}
\lim _{R_{\varepsilon} \rightarrow 0} \int_{\mathcal{L}_{\varepsilon}^{\prime}} \mathfrak{U}_{k l}\left(M_{\circ}, Q_{\circ}\right) \mathfrak{t}_{l}\left(M_{\circ}\right) d s_{M}=0, \\
\lim _{R_{\varepsilon} \rightarrow 0} \int_{\mathcal{L}_{\varepsilon}^{\prime}} \mathfrak{T}_{k l}\left(M, Q_{\circ}\right)\left[\mathfrak{u}_{l}\left(M_{\circ}\right)-\mathfrak{u}_{l}\left(Q_{\circ}\right)\right] d s_{M}=0 .
\end{gathered}
$$

Combining equations (95),...,(96b) for the limit of equation (94) when $R_{\varepsilon} \rightarrow 0$ we obtain

$$
c_{k l}\left(Q_{\circ}\right) \mathfrak{u}_{l}\left(Q_{\circ}\right)=\oint_{\mathcal{L}_{o}} \mathfrak{U}_{k l}\left(M_{\circ}, Q_{\circ}\right) \mathfrak{t}_{l}\left(M_{\circ}\right) d s_{M_{\circ}}-\oint_{\mathcal{L}_{o}} \mathfrak{T}_{k l}\left(M_{\circ}, Q_{\circ}\right) \mathfrak{u}_{l}\left(M_{\circ}\right) d s_{M_{\circ}} .
$$

This result is the second dual SOMIGLIANA formula for inner regions.

REMARK 18.: The integrals in (97) are to be taken in CAUCHY principal value. 
REMARK 19.: Since at a point of the contour $\mathcal{L}_{o}$ either the dual displacements (stress functions) $\mathfrak{u}_{l}\left(M_{\circ}\right)$ or the dual stresses (displacement derivatives) $\mathfrak{t}_{l}\left(M_{\circ}\right)$ can be prescribed, the above integral equation is suitable both for determining the missing $\mathfrak{u}_{l}\left(M_{\circ}\right)$ at a point $Q$ where $\mathfrak{t}_{l}\left(M_{\circ}\right)$ is known, and for determining the missing $\mathfrak{t}_{l}\left(M_{\circ}\right)$ at a point $Q$ where $\mathfrak{u}_{l}\left(M_{\circ}\right)$ is known. Given these quantities on the whole boundary $\mathcal{L}_{o}$, we can use the first dual Somigliana formala for computing the field variables (stresses and strains).

3. If $Q \notin\left(A \cup \mathcal{L}_{o}\right)$ then only the integral over $\mathcal{L}_{o}$ remains in (89), and following steps similar to above, we obtain the third dual Somogliana equation:

$$
0=\oint_{\mathcal{L}_{o}} \mathfrak{U}_{k l}\left(M_{\circ}, Q\right) \mathfrak{t}_{l}\left(M_{\circ}\right) d s_{M_{\circ}}-\oint_{\mathcal{L}_{o}} \mathfrak{T}_{k l}\left(M_{\circ}, Q\right) \mathfrak{u}_{l}\left(M_{\circ}\right) d s_{M_{\circ}} .
$$

REMARK 20.: Assume that

$$
\mathfrak{u}_{l}(M, Q)=\left[\stackrel{o}{\mathcal{F}}_{1}\left|\stackrel{o}{\mathcal{F}}_{2}\right| \stackrel{o}{\mathcal{H}}+\stackrel{o}{\mathcal{F}}_{1} r_{2}(M, Q)-\stackrel{o}{\mathcal{F}}_{2} r_{1}(M, Q)\right]
$$

where $\stackrel{o}{\mathcal{H}}, \stackrel{o}{\mathcal{F}}_{1}$ and $\stackrel{o}{\mathcal{F}}_{2}$ arbitrary constants while $r_{1}$ and $r_{2}$ are the coordinates of some point $M$ with respect to the fixed point $Q$, which may coincide with the origin. It can be checked neither stresses nor strains belong to this dual displacement vector. Hence

$$
{ }^{\prime} \mathfrak{D}_{k l} \mathfrak{u}_{l}=\mathfrak{D}_{k l} \mathfrak{u}_{l}=0, \quad \text { and } \quad \mathfrak{t}_{k}=0 .
$$

Using these equations, identity (89) yields

$$
\int_{A^{+}} \mathfrak{u}_{k}\left({ }^{\prime} \mathfrak{D}_{k l}{ }^{*} \mathfrak{u}_{l}\right) d A=\oint_{\mathcal{L}_{o}} \mathfrak{u}_{k}^{*} \mathfrak{t}_{k} d s
$$

Assume that $\stackrel{*}{\mathfrak{u}}$ and $\mathfrak{t}_{k}$ belong to the fundamental solutions. Then a comparison of equations (48) and (88) gives

$$
{ }^{\prime} \mathfrak{D}_{k l} \stackrel{*}{\mathfrak{u}} l_{l}+\delta(M-Q) e_{k}(Q)=0
$$

Recalling that

$$
\stackrel{*}{\mathfrak{u}_{l}}(M, Q)=\mathfrak{U}_{l s}(M, Q) e_{s}(Q), \quad \stackrel{*}{\mathfrak{t}}_{k}(\stackrel{o}{M}, Q)=e_{s}(Q) \mathfrak{T}_{s l}(\stackrel{o}{M}, Q)
$$

and taking equation (102) into account we get from equation (101) that

$$
-\int_{A^{+}} \mathfrak{u}_{k} \delta(M-Q) \delta_{k l} e_{l}(Q) d A=-e_{l}(Q) \delta_{l k} \mathfrak{u}_{k}(Q, Q) \eta(Q)=\oint_{\mathcal{L}_{o}} e_{l}(Q) \mathfrak{T}_{l k}(\stackrel{o}{M}, Q) \mathfrak{u}_{k}(\stackrel{o}{M}) d s_{M}^{o} .
$$

Given the structure of the dual displacements (99), simple manipulations imply

$$
\oint_{\mathcal{L}_{o}}\left[\mathfrak{T}_{l 1}+r_{2} \mathfrak{T}_{l 3}\left|\mathfrak{T}_{l 2}-r_{1} \mathfrak{T}_{l 3}\right| \mathfrak{T}_{l 3}\right] d s_{M}^{o}=-\eta(Q)\left[\delta_{l 1}\left|\delta_{l 2}\right| \delta_{l 3}\right],
$$

where

$$
\eta(Q)=\left\{\begin{array}{lll}
1 & \text { if } & Q \in A^{+} \\
\frac{1}{2} & \text { if } & Q=\stackrel{o}{Q} \in \mathcal{L}_{o} \\
0 & \text { if } & Q \in A^{-}
\end{array}\right.
$$

This result will allow us to compute the strongly singular integrals in a numerical implementation developed for solving boundary value problems in inner regions. 


\subsection{Formulae for the stresses}

Substituting the first dual SomiglianA formula (93) into equations $(6 a, b)$ we obtain the stresses at the inner points $Q$ of region $A_{i}$ :

$$
\begin{aligned}
& \mathfrak{s}_{1}=t_{11}=\oint_{\mathcal{L}_{\circ}} \underbrace{\left.\left[\mathfrak{U}_{1 l}\left(M_{\circ}, Q\right)\right)^{Q} \stackrel{\partial}{2}_{2}\right]}_{S_{1 l}} \mathfrak{t}_{l}\left(M_{\circ}\right) d s_{M_{\circ}}-\oint_{\mathcal{L}_{o}} \underbrace{\left[\mathfrak{T}_{l 1}\left(M_{\circ}, Q\right) \stackrel{Q}{\partial}_{2}\right]}_{-D_{1 l}} \mathfrak{u}_{l}\left(M_{\circ}\right) d s_{M_{\circ}} \\
& \mathfrak{s}_{2}=t_{12}=\oint_{\mathcal{L}_{o}} \underbrace{\left[\mathfrak{U}_{2 l}\left(M_{\circ}, Q\right) \stackrel{Q}{\partial_{2}}\right]}_{S_{2 l}} \mathfrak{t}_{l}\left(M_{\circ}\right) d s_{M_{\circ}}-\oint_{\mathcal{L}_{o}} \underbrace{\left[\mathfrak{T}_{2 l}\left(M_{\circ}, Q\right) \stackrel{Q}{\partial}_{2}\right]}_{-D_{2 l}} \mathfrak{u}_{l}\left(M_{\circ}\right) d s_{M_{\circ}} \\
& \mathfrak{s}_{3}=t_{21}=-\oint_{\mathcal{L}_{o}} \underbrace{\left[\mathfrak{U}_{1 l}\left(M_{\circ}, Q\right) \stackrel{Q}{\partial}_{1}\right]}_{-S_{3 l}} \mathfrak{t}_{l}\left(M_{\circ}\right) d s_{M_{\circ}}+\oint_{\mathcal{L}_{o}} \underbrace{\left[\mathfrak{T}_{1 l}\left(M_{\circ}, Q\right)^{Q} \stackrel{\partial}{1}_{1}\right]}_{D_{3 l}} \mathfrak{u}_{l}\left({ }_{\circ} M\right) d s_{M_{\circ}} \\
& \mathfrak{s}_{4}=t_{22}=-\oint_{\mathcal{L}_{\circ}} \underbrace{\left[\mathfrak{U}_{2 l}\left(M_{\circ}, Q\right) \partial_{1}^{Q}\right]}_{-S_{4 l}} \mathfrak{t}_{l}\left(M_{\circ}\right) d s_{M_{\circ}}+\oint_{\mathcal{L}_{\circ}} \underbrace{\left[\mathfrak{T}_{2 l}\left(M_{\circ}, Q\right) \partial_{1}\right]}_{D_{4 l}} \mathfrak{u}_{l}\left(M_{\circ}\right) d s_{M_{\circ}} \\
& \mathfrak{m}_{1}=\mu_{13}=\oint_{\mathcal{L}_{o}} \underbrace{\left[\mathfrak{U}_{3 l}\left(M_{\circ}, Q\right) \stackrel{Q}{\partial_{2}}\right]}_{M_{1 l}} \mathfrak{t}_{l}\left(M_{\circ}\right) d s_{M_{\circ}}-\oint_{\mathcal{L}_{\circ}} \underbrace{\left[\mathfrak{T}_{3 l}\left(M_{\circ}, Q\right) \stackrel{Q}{\partial}_{2}\right]}_{N_{1 l}} \mathfrak{u}_{l}\left(M_{\circ}\right) d s_{M_{\circ}}- \\
& -\oint_{\mathcal{L}_{o}}\left[\mathfrak{U}_{1 l}\left(M_{\circ}, Q\right)\right] \mathfrak{t}_{l}\left(M_{\circ}\right) d s_{M_{\circ}}+\oint_{\mathcal{L}_{o}}\left[\mathfrak{T}_{1 l}\left(M_{\circ}, Q\right)\right]\left(M_{\circ}\right) \mathfrak{u}_{l} d s_{M_{\circ}} \\
& \mathfrak{m}_{2}=\mu_{23}=-\oint_{\mathcal{L}_{\circ}} \underbrace{\left[\mathfrak{U}_{3 l}\left(M_{\circ}, Q\right) \stackrel{Q}{\partial}_{1}\right]}_{-M_{2 l}} \mathfrak{t}_{l}\left(M_{\circ}\right) d s_{M_{\circ}}+\oint_{\mathcal{L}_{o}} \underbrace{\left[\mathfrak{T}_{3 l}\left(M_{\circ}, Q\right) \stackrel{Q}{\partial}_{1}\right]}_{-N_{2 l}} \mathfrak{u}_{l}\left(M_{\circ}\right) d s_{M_{\circ}}- \\
& -\oint_{\mathcal{L}_{o}}\left[\mathfrak{U}_{2 l}\left(M_{\circ}, Q\right)\right] \mathfrak{t}_{l}\left(M_{\circ}\right) d s_{M_{\circ}}+\oint_{\mathcal{L}_{o}}\left[\mathfrak{T}_{2 l}\left(M_{\circ}, Q\right)\right] \mathfrak{u}_{l}\left(M_{\circ}\right) d s_{M_{\circ}}
\end{aligned}
$$

or in a more concise form

$$
\mathfrak{s}_{K}(Q)=\oint_{\mathcal{L}_{o}} S_{K l}\left(M_{\circ}, Q\right) \mathfrak{t}_{l}\left(M_{\circ}\right) d s_{M_{\circ}}+\oint_{\mathcal{L}_{o}} D_{K l}\left(M_{\circ}, Q\right) \mathfrak{u}_{l}\left(M_{\circ}\right) d s_{M_{\circ}}, \quad K=1, \ldots, 4
$$

and

$$
\begin{aligned}
\mathfrak{m}_{\kappa}(Q) & =\oint_{\mathcal{L}_{o}} M_{\kappa l}\left(M_{\circ}, Q\right) \mathfrak{t}_{l}\left(M_{\circ}\right) d s_{M_{\circ}}-\oint_{\mathcal{L}_{o}} N_{\kappa l}\left(M_{\circ}, Q\right) \mathfrak{u}_{l}\left(M_{\circ}\right) d s_{M_{\circ}}- \\
& -\oint_{\mathcal{L}_{o}}\left[\mathfrak{U}_{\kappa l}\left(M_{\circ}, Q\right)\right] \mathfrak{t}_{l}\left(M_{\circ}\right) d s_{M_{\circ}}+\oint_{\mathcal{L}_{o}}\left[\mathfrak{T}_{\kappa l}\left(M_{\circ}, Q\right)\right] \mathfrak{u}_{l}\left(M_{\circ}\right) d s_{M_{\circ}}, \quad \kappa=1,2 .
\end{aligned}
$$

Matrices $S_{K l}\left(M_{\circ}, Q\right)$ and $M_{\kappa l}\left(M_{\circ}, Q\right)$ are all presented in Appendix A. Since the formulae for $D_{K l}\left(M_{\circ}, Q\right)$ and $N_{\kappa l}\left(M_{\circ}, Q\right)$ are quite long we have not presented them in Appendix A. 


\section{Dual Somigliana formulae for outer regions}

\subsection{Stresses at infinity}

Let $A^{-}$denote the part of the plane that lies outside the contour $\mathcal{L}_{o}$ - see Figure 4 . Assume that the force stresses and couple stresses are constants at infinity. These are denoted by

$$
t_{11}(\infty), t_{12}(\infty), t_{21}(\infty), t_{22}(\infty) \quad \text { and } \quad \mu_{13}(\infty), \mu_{23}(\infty)
$$

By assumption the body forces and couples $b_{\rho}$ and $c_{3}$ vanish at infinity.

Since the couple stresses $\mu_{\nu 3}(\infty)$ are constants at infinity it follows from the equilibrium equation $(3)_{2}$ that the force stress tensor meets the symmetry condition

$$
\epsilon_{3 \nu \rho} t_{\nu \rho}(\infty)=0
$$

Consider the stress functions

$$
\mathcal{F}_{\rho}=\varepsilon_{\pi \nu 3} t_{\rho \pi}(\infty) \xi_{\nu}+c_{\rho}(\infty)
$$

and

$$
\mathcal{H}=\mu_{13}(\infty) \xi_{2}-\mu_{23}(\infty) \xi_{1}+t_{11}(\infty) \frac{\xi_{2}^{2}}{2}-t_{12}(\infty) \xi_{1} \xi_{2}+t_{22}(\infty) \frac{\xi_{1}^{2}}{2}+c_{1}(\infty) \xi_{2}-c_{2}(\infty) \xi_{1}
$$

where $c_{\rho}(\infty)$ is an arbitrary constant. If we substitute these stress functions into $(6 a, b)$, in which we neglect the particular solutions since the body forces and couples are assumed to be zero, it follows that the stresses are constant on the entire plane. It is easy to see that the stress state

$$
t_{11}(\infty), t_{12}(\infty), t_{21}(\infty), t_{22}(\infty) \quad \text { and } \quad \mu_{13}(\infty), \mu_{23}(\infty)
$$

should be an elastic state of the whole plane (or of any subregion). As a result, the strains that belong to the above stress state should meet the dual balance equations (8) (the compatibility equations).

Since the couple stress tensor $\mu_{\nu 3}$ is constant it follows from the HoOKE law (7b) that the curvature twist tensor $\kappa_{\nu 3}$ is also constant, i.e., it satisfies the dual balance equation $(8)_{1}$.

If the force stress tensor $t_{\pi \rho}$ is constant then according to the HoOKE law (7a) so is the strain tensor $\gamma_{\pi \rho}$. The dual balance equation $(8)_{2}$ then implies that

$$
D_{\rho}=\epsilon_{3 \nu \pi} \gamma_{\pi \rho} \partial_{\nu}-\kappa_{\rho 3}=-\kappa_{\rho 3} \neq 0
$$

which means that the second dual balance equation is satisfied if and only if $\kappa_{\rho 3}=0$ or, equivalently given the HOOKE law (2b), if

$$
\mu_{\nu 3}(\infty)=0
$$

It then follows that the stress state at infinity is described by the stress functions

$$
\mathcal{F}_{1}=\tilde{\mathfrak{u}}_{1}(Q)=\xi_{2} t_{11}(\infty)-\xi_{1} t_{12}(\infty)+c_{1}(\infty), \quad \mathcal{F}_{2}=\tilde{\mathfrak{u}}_{2}(Q)=\xi_{2} t_{21}(\infty)-\xi_{1} t_{22}(\infty)+c_{2}(\infty)
$$

and

$$
\mathcal{H}=\tilde{\mathfrak{u}}_{3}(Q)=t_{11}(\infty) \frac{\xi_{2}^{2}}{2}-t_{12}(\infty) \xi_{1} \xi_{2}+t_{22}(\infty) \frac{\xi_{1}^{2}}{2}+c_{1}(\infty) \xi_{2}-c_{2}(\infty) \xi_{1}
$$

which provide an elastic state on the whole plane (or on any part of it). 


\subsection{Derivation of the dual Somigliana formulae for outer regions}

Figure 4 depicts a triple connected region $A^{\prime}$ bounded by the contours $\mathcal{L}_{o}, \mathcal{L}_{\varepsilon}$ and the circle $\mathcal{L}_{R}$ with radius ${ }_{e} R$ and center $O$. Here $\mathcal{L}_{\varepsilon}$ is the contour of the neighborhood $A_{\varepsilon}$ of $Q$ with radius $R_{\varepsilon}$ while ${ }_{e} R$ is sufficiently large so that the region bounded by $\mathcal{L}_{R}$ covers both $\mathcal{L}_{0}$, and $\mathcal{L}_{\varepsilon}$. If ${ }_{e} R \rightarrow \infty$ and $R_{\varepsilon} \rightarrow 0$ then clearly $A^{\prime} \rightarrow A^{-}$.

Let $\mathfrak{u}_{k}(M)$ and $\mathfrak{u}_{k}(M)$ be sufficiently smooth elastic states (dual displacements) on $A^{-}$. The corresponding dual stresses on the contour are denoted by $\mathfrak{t}_{k}$ and $\mathfrak{t}_{k}$, respectively.

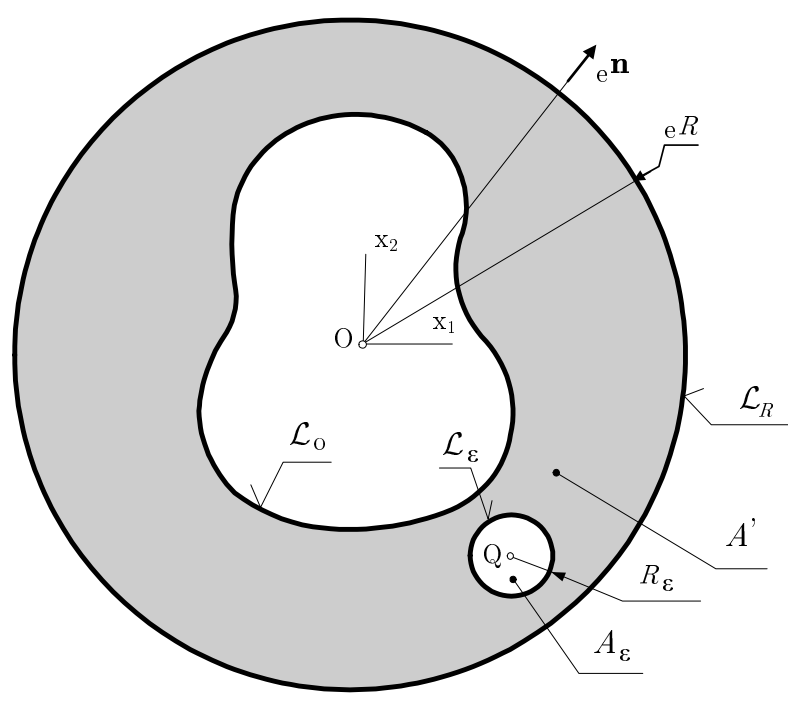

Figure 4: Exterior region, $Q$ is an inner point

Equation

$$
\begin{aligned}
& \int_{A^{\prime}}\left[\mathfrak{u}_{k}(M)\left(\stackrel{M}{\mathfrak{D}_{k l}}{ }^{*} \mathfrak{u}_{l}(M)\right)-\mathfrak{u}_{k}(M)\left(\stackrel{M}{\mathfrak{D}_{k l}} \mathfrak{u}_{l}(M)\right)\right] \mathrm{d} A_{M}= \\
& =\oint_{\mathcal{L}_{o}}\left[\mathfrak{u}_{l}(\stackrel{o}{M}) \mathfrak{\mathfrak { t }}_{l}(\stackrel{o}{M})-\stackrel{*}{\mathfrak{u}_{l}}(\stackrel{o}{M}) \mathfrak{t}_{l}(\stackrel{o}{M})\right] \mathrm{d} s_{M}^{o}+\oint_{\mathcal{L}_{\varepsilon}}\left[\mathfrak{u}_{l}(\stackrel{o}{M}) \stackrel{*}{\mathfrak{t}_{l}}(\stackrel{o}{M})-\stackrel{*}{\mathfrak{u}_{l}}(\stackrel{o}{M}) \mathfrak{t}_{l}(\stackrel{o}{M})\right] \mathrm{d} s_{M}^{o} \\
& +\oint_{\mathcal{L}_{R}}\left[\mathfrak{u}_{l}(\stackrel{o}{M}) \stackrel{*}{\mathfrak{t}_{l}}(\stackrel{o}{M})-\stackrel{*}{\mathfrak{u}}_{l}(\stackrel{o}{M}) \mathfrak{t}_{\lambda}(\stackrel{o}{M})\right] \mathrm{d} s_{M}^{o}
\end{aligned}
$$

is the dual Somigliana identity (89) when it is applied to the triple connected region $A_{e}^{\prime}$. Observe that $M$ over a letter means that the corresponding derivatives are taken with respect to the coordinates of $M$. Let again

$$
\stackrel{*}{\mathfrak{u}_{l}}(Q)=e_{k}(Q) \mathfrak{U}_{k l}(M, Q) \quad \text { and } \quad \stackrel{*}{\mathfrak{t}_{l}}(\stackrel{o}{M})=e_{k}(Q) \mathcal{T}_{k l}(\stackrel{o}{M}, Q)
$$

which provide a non singular elastic state of the plane in $A^{\prime}$. We regard $\mathfrak{u}_{l}(M)$ as a different elastic state in the region $A^{-}$. Further we assume that $\mathfrak{u}_{l}(M)$ has the the following far field pattern (asymptotic behavior)

$$
\mathfrak{u}_{k}=\tilde{\mathfrak{u}}_{k}
$$

Without loss of generality, assume that the origin is an inner point of the region $A^{+}$. We need to consider three cases depending on the location of the point $Q$ - Figure 4 represents the first one.

1. If $Q \in A^{-}$then region $A^{\prime}$ is the subject of our investigation. Substituting the above quantities into the SOMIGLIANA identity (114) and then noting that the surface integrals vanish and omitting 
$e_{k}(Q)$ we have

$$
\begin{aligned}
& \oint_{\mathcal{L}_{o}}\left[\mathfrak{T}_{k l}\left(M_{\circ}, Q\right) \mathfrak{u}_{l}\left(M_{\circ}\right)-\mathfrak{U}_{k l}\left(M_{\circ}, Q\right) \mathfrak{t}_{l}\left(M_{\circ}\right)\right] d s_{M_{\circ}}+ \\
& \quad+\oint_{\mathcal{L}_{\varepsilon}}\left[\mathfrak{T}_{k l}\left(M_{\circ}, Q\right) \mathfrak{u}_{l}\left(M_{\circ}\right)-\mathfrak{U}_{k l}\left(M_{\circ}, Q\right) \mathfrak{t}_{l}\left(M_{\circ}\right)\right] d s_{M_{\circ}}+ \\
& \quad+\oint_{\mathcal{L}_{R}}\left[\mathfrak{T}_{k l}\left(M_{\circ}, Q\right) \mathfrak{u}_{l}\left(M_{\circ}\right)-\mathfrak{U}_{k l}\left(M_{\circ}, Q\right) \mathfrak{t}_{l}\left(M_{\circ}\right)\right] d s_{M_{\circ}}=0 .
\end{aligned}
$$

To compute the limit of the first two integrals as $R_{\varepsilon} \longrightarrow 0$ and ${ }_{e} R \longrightarrow \infty$, note that they coincide with (93) if in the latter all terms are moved to the left side. Consequently

$$
\oint_{\mathcal{L}_{o}} \cdots+\lim _{R_{\varepsilon} \longrightarrow 0} \oint_{\mathcal{L}_{\varepsilon}} \cdots=\mathfrak{u}_{k}(Q)+\oint_{\mathcal{L}_{o}}\left[\mathfrak{T}_{k l}\left(M_{\circ}, Q\right) \mathfrak{u}_{l}\left(M_{\circ}\right)-\mathfrak{U}_{k l}\left(M_{\circ}, Q\right) \mathfrak{t}_{l}\left(M_{\circ}\right)\right] d s_{M_{\circ}} .
$$

We now need to determine the limit of the third integral denoted by $I_{R}$ :

$$
\lim _{e R \longrightarrow \infty}\{\underbrace{\oint_{\mathcal{L}_{R}}\left[\mathfrak{U}_{k l}\left(M_{\circ}, Q\right) \mathfrak{t}_{l}\left(M_{\circ}\right)-\mathfrak{T}_{k l}\left(M_{\circ}, Q\right) \mathfrak{u}_{l}\left(M_{\circ}\right)\right] d s_{M_{\circ}}}_{I_{R}}\} .
$$

In this equation

(a) $I_{R}$ coincides with the right side of the first dual Somigliana formula (93) which is valid since $Q$ is an inner point of the region;

(b) if ${ }_{e} R \longrightarrow \infty$ then $\mathfrak{u}_{l}$ and $\mathfrak{t}_{l}$ on the circle belong to the elastic state of the plane for which the force stresses are constant and the couple stresses vanish everywhere.

Therefore we get

$$
\lim _{e R \longrightarrow \infty} I_{R}=\tilde{\mathfrak{u}}_{k}(Q) .
$$

Comparing equations (115), (116) and (118) yields the first dual SOMIGLIANA formula for exterior regions:

$$
\mathfrak{u}_{k}(Q)=\tilde{\mathfrak{u}}_{k}(Q)+\oint_{\mathcal{L}_{\circ}} \mathfrak{U}_{k l}\left(M_{\circ}, Q\right) \mathfrak{t}_{l}\left(M_{\circ}\right) d s_{M_{\circ}}-\oint_{\mathcal{L}_{o}} \mathfrak{T}_{k l}\left(M_{\circ}, Q\right) \mathfrak{u}_{l}\left(M_{\circ}\right) d s_{M_{\circ}}
$$

2. If $Q=\stackrel{o}{M}$ is on $\mathcal{L}_{o}$, nothing changes concerning the limit of the integral taken on $\mathcal{L}_{R}$. Consequently

$$
\begin{aligned}
c_{k l}\left(Q_{\circ}\right) \mathfrak{u}_{l}\left(Q_{\circ}\right)=\tilde{\mathfrak{u}}_{k}\left(Q_{\circ}\right)+\oint_{\mathcal{L}_{o}} \mathfrak{U}_{k l}\left(M_{\circ}, Q_{\circ}\right) \mathfrak{t}_{l}\left(M_{\circ}\right) d s_{M_{\circ}}- & \\
& -\oint_{\mathcal{L}_{\circ}} \mathfrak{T}_{k l}\left(M_{\circ}, Q_{\circ}\right) \mathfrak{u}_{l}\left(M_{\circ}\right) d s_{M_{\circ}} .
\end{aligned}
$$

REMARK 21.: Equation (120) is an integral equation for the unknowns $\mathfrak{u}_{l}\left(M_{\circ}\right) M_{\circ} \in \mathcal{L}_{u}$ and $\mathfrak{t}_{l}\left(M_{\circ}\right) M_{\circ} \in \mathcal{L}_{t}$. This is the integral equation of the direct method.

REMARK 22.: We do not detail the derivations leading to (120). This is because the integrals taken on $\mathcal{L}_{R}$ are computed exactly the same way as before, and the other terms can be obtained in analogously to the integral equation (97) we set up for inner regions. 
3. If $Q$ is inside the contour $\mathcal{L}_{o}$, i.e., it lies in the region $A^{+}$, then it is easy to show that

$$
0=\tilde{\mathfrak{u}}_{k}(Q)+\oint_{\mathcal{L}_{o}} \mathfrak{U}_{k l}\left(M_{\circ}, Q\right) \mathfrak{t}_{l}\left(M_{\circ}\right) d s_{M_{\circ}}-\oint_{\mathcal{L}_{o}} \mathfrak{T}_{k l}\left(M_{\circ}, Q\right) \mathfrak{u}_{l}\left(M_{\circ}\right) d s_{M_{\circ}} .
$$

which is the third SOMIGLIANA formula for exterior regions.

REMARK 23.: Let us assume again recalling Remark 20 that $\mathfrak{u}_{k}$ is taken from equation (99) - see Remark 20 for details - and apply first relation (101) to region $A_{R}$ in Figure $4\left(\mathcal{L}_{R}\right.$ and $A_{R}$ should stand for $\mathcal{L}_{o}$ and $A^{+}$in the relation we have referred to). Further let $Q$ be an inner point of region $A_{R}$. Assume

again that $\mathfrak{\mathfrak { u }}_{l}$ and $\mathfrak{\mathfrak { t }}_{k}$ belong to the fundamental solutions. Then repeating the line of thought resulting in equation (103) we obtain

$$
\int_{A_{R}} \mathfrak{u}_{k}\left(' \mathfrak{D}_{k l}^{*} \mathfrak{u}_{l}\right) d A=\oint_{\mathcal{L}_{R}} \stackrel{*}{\mathfrak{u}_{k}} \mathfrak{t}_{k} d s=-\mathfrak{u}_{l}(Q, Q) e_{l}(Q)
$$

Next apply (101) to region $A^{\prime}$ in Figure 4. We get

$$
\oint_{\mathcal{L}_{o}} \stackrel{*}{\mathfrak{u}_{k} \mathfrak{t}_{k}} d s+\oint_{\mathcal{L}_{R}} \stackrel{*}{\mathfrak{u}_{k}} \mathfrak{t}_{k} d s=\int_{A^{\prime}} \mathfrak{u}_{k}\left({ }^{\prime} \mathfrak{D}_{k l}{ }^{*} \mathfrak{u}_{l}\right) d A
$$

where using (103) we obtain

$$
\int_{A^{\prime}} \mathfrak{u}_{k}\left({ }^{\prime} \mathfrak{D}_{k l} \stackrel{*}{\mathfrak{u}} l_{l}\right) d A=-\eta(Q) e_{l}(Q)\left[\stackrel{o}{\mathcal{F}_{1}} \delta_{l 1}\left|\stackrel{o}{\mathcal{F}}_{2} \delta_{l 2}\right| \stackrel{o}{\mathcal{H}} \delta_{l 3}\right]
$$

while the integral on contour $\mathcal{L}_{R}$ can be taken from equation (122). Consequently the following equation

$$
\oint_{\mathcal{L}_{o}} \mathfrak{u}_{k} \mathfrak{t}_{k} d s-\mathfrak{u}_{l}(Q, Q) e_{l}(Q)=-\eta(Q) e_{l}(Q)\left[\stackrel{o}{\mathcal{F}_{1}} \delta_{l 1}\left|\stackrel{o}{\mathcal{F}}_{2} \delta_{l 2}\right| \stackrel{o}{\mathcal{H}} \delta_{l 3}\right]
$$

holds independently of $R$, and hence for $R \rightarrow \infty$ as well. Recalling the definition of $\mathfrak{u}_{k}$ we arrive at the final form of the above equation

$$
\oint_{\mathcal{L}_{o}}\left[\mathfrak{T}_{l 1}+r_{2} \mathfrak{T}_{l 3}\left|\mathfrak{T}_{l 2}-r_{1} \mathfrak{T}_{l 3}\right| \mathfrak{T}_{l 3}\right] d s_{M}-\left[\delta_{l 1}\left|\delta_{l 2}\right| \delta_{l 3}\right]=-\eta(Q)\left[\delta_{l 1}\left|\delta_{l 2}\right| \delta_{l 3}\right]
$$

where

$$
\eta(Q)=\left\{\begin{array}{lll}
1 & \text { if } & Q \in A^{-} \\
\frac{1}{2} & \text { if } & Q=\stackrel{o}{Q} \in \mathcal{L}_{o} \\
0 & \text { if } & Q \in A^{+}
\end{array}\right.
$$

This result will allow us to compute the strongly singular integrals in a numerical implementation set up for exterior regions.

\section{Calculations of the stresses on the boundary}

After solving the integral equations of the direct method (equation (97) for inner regions, equation (120) for exterior regions) we know the dual displacement vector $\mathfrak{u}_{l}$ as well as the dual stresses $\mathfrak{t}_{l}$, which can be given in terms of displacements derivatives with respect to the arc coordinate, on the contour.

The next question is how to determine the stresses on the contour in terms of these quantities. 
Using equations (14b), (14c) and taking (4b) into account we have

$$
\begin{gathered}
\frac{d \mathcal{F}_{\rho}}{d s}=n_{1} t_{1 \rho}+n_{2} t_{2 \rho}, \\
\frac{d \mathcal{H}}{d s}-n_{\rho} \mathcal{F}_{\rho}=n_{1} \mu_{13}+n_{2} \mu_{23} .
\end{gathered}
$$

The above equations are to be supplemented by the definition of the dual stresses (75):

$$
\mathfrak{t}_{\rho}=\tau_{\pi} \gamma_{\pi \rho} \quad \text { and } \quad \mathfrak{t}_{3}=\tau_{\pi} \gamma_{\pi 3}
$$

The left sides of equations (126), ..,(128) are all known as soon as we have solved the integral equations of the direct method. Since the strains can always be given in terms of stresses via the HoOKE law (7a) and $(7 b)$ the right hand sides contain the unknown force and couple stresses

$$
t_{11}, t_{12}, t_{21} t_{22}, \mu_{12} \text { and } \mu_{23} \text {. }
$$

If we consider equations $(126), \ldots,(128)$ in the coordinate system $(x y z)$ by substituting the relations $n_{x}=\tau_{y}$ and $n_{y}=-\tau_{x}$ into (128) we obtain the system of linear equations

$$
\begin{gathered}
\frac{d \mathfrak{u}_{x}}{d s}=n_{x} t_{x x}+n_{y} t_{y x}, \\
\frac{d \mathfrak{u}_{y}}{d s}=n_{x} t_{x y}+n_{y} t_{y y}, \\
\frac{d \mathfrak{u}_{3}}{d s}-n_{x} \mathcal{F}_{x}-n_{y} \mathcal{F}_{y}=n_{x} \mu_{x z}+n_{y} \mu_{y z}, \\
\mathfrak{t}_{x}=-n_{y} \gamma_{x x}+n_{x} \gamma_{y x}=-n_{y}\left[\frac{1}{2 \mu} t_{x x}-\frac{\nu}{2 \mu}\left(t_{x x}+t_{y y}\right)\right]+n_{x}\left[\frac{1}{2 \mu} \frac{t_{y x}+t_{x y}}{2}+\frac{1}{2 \alpha} \frac{t_{y x}-t_{x y}}{2}\right], \\
\mathfrak{t}_{y}=-n_{y} \gamma_{x y}+n_{x} \gamma_{y y}=-n_{y}\left[\frac{1}{2 \mu} \frac{t_{x y}+t_{y x}}{2}+\frac{1}{2 \alpha} \frac{t_{x y}-t_{y x}}{2}\right]+n_{x}\left[\frac{1}{2 \mu} t_{y y}-\frac{\nu}{2 \mu}\left(t_{x x}+t_{y y}\right)\right], \\
\mathfrak{t}_{z}=-n_{y} \kappa_{x z}+n_{x} \kappa_{y z}=\frac{1}{\gamma+\varepsilon}\left(-n_{y} \mu_{x z}+n_{x} \mu_{y z}\right)
\end{gathered}
$$

which can be solved for the stresses

$$
t_{x x}, t_{x y}, t_{y x} t_{y y}, \mu_{x z} \text { and } \mu_{y z} .
$$

For completeness we rewrite this equation system into a matrix form:

$$
\begin{array}{r}
{\left[\begin{array}{cccccc}
n_{x} & 0 & n_{y} & 0 & 0 & 0 \\
0 & n_{x} & 0 & n_{y} & 0 & 0 \\
0 & 0 & 0 & 0 & n_{x} & n_{y} \\
-\frac{1-\nu}{2 \mu} n_{y} & \frac{\alpha-\mu}{4 \mu \alpha} n_{x} & \frac{\alpha+\mu}{4 \mu \alpha} n_{x} & \frac{\nu}{2 \mu} n_{y} & 0 & 0 \\
-\frac{\nu}{2 \mu} n_{x} & -\frac{\alpha+\mu}{4 \mu \alpha} n_{y} & -\frac{\alpha-\mu}{4 \mu \alpha} n_{y} & \frac{1-\nu}{2 \mu} n_{x} & 0 & 0 \\
0 & 0 & 0 & 0 & -\frac{1}{\gamma+\varepsilon} n_{y} & \frac{1}{\gamma+\varepsilon} n_{x}
\end{array}\right]\left[\begin{array}{c}
t_{x x} \\
t_{x y} \\
t_{y x} \\
t_{y y} \\
\mu_{x z} \\
\mu_{y z}
\end{array}\right]=} \\
\\
=\left[\begin{array}{c}
\frac{d \mathfrak{u}_{x}}{d s} \\
\frac{d \mathfrak{u}_{y}}{d s} \\
\frac{d \mathfrak{u}_{3}}{d s}-n_{x} \mathcal{F}_{x}-n_{y} \mathcal{F}_{y} \\
\mathfrak{t}_{x} \\
\mathfrak{t}_{y} \\
\mathfrak{t}_{z}
\end{array}\right]
\end{array}
$$




\section{Solution algorithm and numerical examples}

\subsection{The algorithm}

To solve the integral equation (120), we divide the contour $\mathcal{L}_{o}$ into $n_{b e}$ arcs, which are the boundary elements. The endpoints and midpoint of an element are, in many cases, the nodes on the element their number is denoted by $n_{e n}$ (number of element nodes). The coordinates of an element as well as the functions $\mathfrak{u}_{l}\left(M_{\circ}\right)$ and $\mathfrak{t}_{l}\left(M_{\circ}\right)$ over an element are approximated by quadratic polynomials based on the nodal points. The order of the polynomial depends on the number of element nodes.

A quadratic approximation over an element might also be based on a different choice for the location of the nodal points. The approximation is partly discontinuous if the [first] \{last\} nodal point is inside the element, the [last] \{first $\}$ nodal point is an endpoint of the element and there is a third nodal point between the two.

The nodal points are numbered locally increasing in the positive direction of the arc coordinate $s$ and the node numbers take the values 1,2 and 3 .

The global node numbering differs from the local one. Let $Q_{i}$ be the $i$-th node on the contour $\mathcal{L}_{o}: i=1,2, \ldots, n_{b n}$, where $n_{b n}$ is the number of the boundary nodes on the contour. These are also numbered starting form an arbitrary nodal point of the contour and increasing in the positive direction.

In the computations we apply continuous (or partly discontinuous) quadratic shape functions. The arc coordinate on the element is mapped onto the interval $\eta \in[-1,1]$ while the shape functions $N^{i}(\eta)$ are Lagrange polynomials given in terms of the nodal coordinates $\eta^{1}<\eta^{2}<\eta^{3} ; \eta_{i} \in[-1,1] i=1,2,3$ :

$$
\begin{gathered}
N^{1}(\eta)=\frac{1}{\left(\eta^{1}-\eta^{2}\right)\left(\eta^{1}-\eta^{3}\right)}\left(\eta-\eta^{2}\right)\left(\eta-\eta^{3}\right), \\
N^{2}(\eta)=\frac{1}{\left(\eta^{2}-\eta^{3}\right)\left(\eta^{2}-\eta^{1}\right)}\left(\eta-\eta^{3}\right)\left(\eta-\eta^{1}\right), \quad N^{3}(\eta)=\frac{1}{\left(\eta^{3}-\eta^{1}\right)\left(\eta^{3}-\eta^{2}\right)}\left(\eta-\eta^{1}\right)\left(\eta-\eta^{2}\right)
\end{gathered}
$$

where $\eta^{1}=-1$ and $-1<\eta^{2}<\eta^{3}<1$ if there is a discontinuity at the point $\eta=1$ while $\eta^{3}=1$ and $-1<\eta^{1}<\eta^{2}<1$ if a discontinuity occurs at the point $\eta=-1$.

Let $e_{\lambda}^{q}, \stackrel{e}{\mathfrak{u}}_{l}^{q}$ and $\mathfrak{\mathfrak { t }}_{l}^{e}$ be the nodal coordinates, the dual displacements and the dual stresses at the $q$-th nodal point of the element $e$. Then

$$
\stackrel{e}{x_{\lambda}}=\sum_{q=1}^{n_{e n}} N^{q}(\eta) \stackrel{e}{x_{\lambda}^{q}}, \quad \stackrel{e}{\mathfrak{u}_{l}}=\sum_{k=1}^{n_{e n}} N^{q}(\eta) \stackrel{e_{\mathfrak{u}}^{q}}{\mathfrak{u}_{l}} \quad \text { and } \quad \stackrel{e}{\mathfrak{t}_{l}}=\sum_{k=1}^{n_{e n}} N^{q}(\eta) \stackrel{e_{\mathfrak{t}}^{q}}{\mathfrak{l}_{l}}
$$

are the approximations of the contour, the dual displacements and the dual stresses on the $e$-th element.

Using equation $(131)_{1}$ and the definition of the shape functions (130) we can set up a closed form relation between $d s$ and $d \eta$ :

$$
\mathrm{d} s=\underbrace{\sqrt{\left(\frac{\mathrm{d} x_{1}}{\mathrm{~d} \eta}\right)^{2}+\left(\frac{\mathrm{d} x_{2}}{\mathrm{~d} \eta}\right)^{2}}}_{J(\eta)} \mathrm{d} \eta=J(\eta) \mathrm{d} \eta .
$$

The equation that provides $J(\eta)$ is not detailed here. 


\subsection{The equation system to be solved}

Substituting approximations (131) into integral equation (97) and integrating element by element we obtain

$$
\begin{aligned}
c_{k l}\left(Q_{\circ}\right) \mathfrak{u}_{l}\left(Q_{\circ}\right)=\sum_{e=1}^{n_{b e}} \int_{\mathcal{L}_{o}} \mathfrak{U}_{k l}\left(\eta, Q_{\circ}\right) \sum_{q=1}^{n_{e n}} N^{q}(\eta) J(\eta) \mathrm{d} \eta \stackrel{e}{\mathfrak{t}}_{l}^{k}- \\
\quad-\sum_{e=1}^{n_{b e}} \int_{\mathcal{L}_{o}} \mathfrak{T}_{k l}\left(\eta, Q_{\circ}\right) \sum_{k=1}^{n_{e n}} N^{q}(\eta) J(\eta) \mathrm{d} \eta \stackrel{e_{\mathfrak{u}}^{q}}{q}, \quad Q=Q_{\circ} \in \mathcal{L}_{o} .
\end{aligned}
$$

Let

$$
\mathbf{u}_{j}=\left[\begin{array}{c}
\mathfrak{u}_{1}^{j} \\
\mathfrak{u}_{2}^{j} \\
\mathfrak{u}_{3}^{j}
\end{array}\right] \quad \text { and } \quad \mathbf{t}_{j}=\left[\begin{array}{c}
\mathfrak{t}_{1}^{j} \\
\mathfrak{t}_{2}^{j} \\
\mathfrak{t}_{3}^{j}
\end{array}\right], \quad j=1, \ldots, n_{b n}
$$

be the matrices of the dual displacements and stresses at the nodal point $j$. For the whole contour equations

$$
\mathbf{u}^{T}=[\underbrace{\mathfrak{u}_{1}^{1} \mathfrak{u}_{2}^{1} \mathfrak{u}_{3}^{1}}_{\mathbf{u}_{1}^{T}}|\underbrace{\mathfrak{u}_{1}^{2} \mathfrak{u}_{2}^{2} \mathfrak{u}_{3}^{2}}_{\mathbf{u}_{2}^{T}}| \cdots \mid \underbrace{\mathfrak{u}_{1}^{n_{b n}} \mathfrak{u}_{2}^{n_{b n}} \mathfrak{u}_{3}^{n_{b n}}}_{\mathbf{u}_{n_{b n}}^{T}}]
$$

and

$$
\mathbf{t}^{T}=[\underbrace{\mathfrak{t}_{1}^{1} \mathfrak{t}_{2}^{1} \mathfrak{t}_{3}^{1}}_{\mathbf{u}_{1}^{T}}|\underbrace{\mathfrak{t}_{1}^{2} \mathfrak{t}_{2}^{2} \mathfrak{t}_{3}^{2}}_{\mathbf{u}_{2}^{T}}| \cdots \mid \underbrace{\mathfrak{t}_{1}^{n_{b n}} \mathfrak{t}_{2}^{n_{b n}} \mathfrak{t}_{3}^{n_{b n}}}_{\mathbf{u}_{n_{b n}}^{T}}]
$$

define the matrices of the dual displacements $\mathbf{u}$ and the dual stresses $\mathbf{t}$ where ${ }^{T}$ denotes the transpose of a matrix. The value of the function $a(j, e)$ is the local node number that belongs to the global node number $j$ on element $e$. To simplify later derivations, we introduce the $3 \times 3$ submatrices $\mathbf{h}_{i j}$ and $\mathbf{b}_{i j}$ defined as

$$
\hat{\mathbf{h}}_{i j}=\left[\sum_{e \in j} \int_{\mathcal{L}_{e}} \mathfrak{T}_{k l}\left(Q_{i}, \eta\right) N^{a(j, e)}(\eta) J(\eta) d \eta\right]
$$

and

$$
\mathbf{b}_{i j}=\left[\sum_{e \in j} \int_{\mathcal{L}_{e}} \mathfrak{U}_{k l}\left(Q_{i}, \eta\right) N^{a(j, e)}(\eta) J(\eta) d \eta\right]
$$

in which (a) $Q_{i}$ is the $i$-t nodal point referred to as collocation point; (b) the summation is over those boundary elements having the nodal point $j$ as their common nodal point; and (c) $N^{a(j, e)}(\eta)$ is the $a$-th shape function. Introducing the additional notation

$$
\mathbf{c}_{i i}=\left[c_{\kappa \lambda}\left(Q_{i}\right)\right]
$$

and

$$
\mathbf{h}_{i j}= \begin{cases}\hat{\mathbf{h}}_{i i}+\mathbf{c}_{i i}, & \text { ha } i=j \\ \hat{\mathbf{h}}_{i j}, & \text { ha } i \neq j\end{cases}
$$

and then assuming that $Q_{o}=Q_{i}$, we can rewrite equation (133) as

$$
\left[\begin{array}{llll}
\mathbf{h}_{i 1} & \mathbf{h}_{i 2} & \cdots & \mathbf{h}_{i n_{b n}}
\end{array}\right]\left[\begin{array}{l}
\mathbf{u}_{1} \\
\mathbf{u}_{2} \\
\cdots \\
\mathbf{u}_{n_{b n}}
\end{array}\right]=\left[\begin{array}{llll}
\mathbf{b}_{i 1} & \mathbf{b}_{i 2} & \cdots & \mathbf{b}_{i n_{b n}}
\end{array}\right]\left[\begin{array}{l}
\mathbf{t}_{1} \\
\mathbf{t}_{2} \\
\ldots \\
\mathbf{t}_{n_{b n}}
\end{array}\right], i=1, \ldots, n_{b n}
$$


Unification of the above equations yields

$$
\left[\begin{array}{cccc}
\mathbf{h}_{11} & \mathbf{h}_{12} & \cdots & \mathbf{h}_{1 n_{b n}} \\
\mathbf{h}_{21} & \mathbf{h}_{22} & \cdots & \mathbf{h}_{2 n_{b n}} \\
\ldots \ldots \ldots \ldots \ldots \ldots \ldots & \ldots \ldots \ldots \\
\mathbf{h}_{n_{b n} 1} & \mathbf{h}_{n_{b n} 2} & \cdots & \mathbf{h}_{n_{b n} n_{b n}}
\end{array}\right]\left[\begin{array}{l}
\mathbf{u}_{1} \\
\mathbf{u}_{2} \\
\cdots \\
\mathbf{u}_{n_{b n}}
\end{array}\right]=\left[\begin{array}{cccc}
\mathbf{b}_{11} & \mathbf{b}_{12} & \cdots & \mathbf{b}_{1 n_{b n}} \\
\mathbf{b}_{21} & \mathbf{b}_{22} & \cdots & \mathbf{b}_{2 n_{b n}} \\
\ldots \ldots \ldots \ldots \ldots \ldots \ldots \ldots \ldots \ldots \\
\mathbf{b}_{n_{b n} 1} & \mathbf{b}_{n_{b n} 2} & \cdots & \mathbf{b}_{n_{b n} n_{b n}}
\end{array}\right]\left[\begin{array}{l}
\mathbf{t}_{1} \\
\mathbf{t}_{2} \\
\cdots \\
\mathbf{t}_{n_{b n}}
\end{array}\right],
$$

which can be cast into a more compact form as

$$
\mathbf{H u}=\mathbf{B t} .
$$

In the above equation either $\mathbf{u}_{k}$ or $\mathbf{t}_{k}$ is known from the boundary conditions. Consequently we have as many equations as there are unknowns. After determining the unknown nodal values both $\mathfrak{u}_{l}\left(M_{\circ}\right)$ and $\mathfrak{t}_{l}\left(M_{\circ}\right)$ can be regarded as known functions on the contour $\mathcal{L}_{o}$.

Field variables at inner points are then obtained from equations (93) and (106a,...,f), (107a,b).

Determination of the stresses on the boundary part $\mathcal{L}_{u}$ requires the solution of equations (129).

Determination of the diagonal submatrices $\mathbf{h}_{i i}, i=1, \ldots, n_{b n}$ requires the computation of strongly singular integrals. However we can avoid computing strongly singular integrals if we follow the following procedure.

Let $Q_{i}$ be the collocation point and typeset [the elements of the matrix $\left.\mathbf{h}_{i j}\right]\left\{\right.$ the matrix $\mathbf{h}_{i j}$ in full $\}$ in the following way:

$$
h_{k l}^{i j} \quad \text { and } \quad\left[\begin{array}{lll}
h_{11} & h_{12} & h_{13} \\
h_{21} & h_{22} & h_{23} \\
h_{31} & h_{33} & h_{33}
\end{array}\right]^{i j}, \quad i, j=1, \ldots, n_{b n}, \quad k, l=1,2,3
$$

First assume that $\stackrel{o}{\mathcal{F}}_{1}=\stackrel{o}{\mathcal{F}}_{2}=0$ and $\stackrel{o}{\mathcal{H}}=1$. It follows from Remark 20 - see page 20 for details - that no stresses and strains belong to the dual displacements

$$
\mathfrak{u}_{l}\left(M, Q_{i}\right)=[0|0| 1]
$$

obtained from equation (99). Substituting the above dual displacements into equation (141) we have

$$
\sum_{j=1}^{j=n_{b n}}\left[\begin{array}{lll}
h_{11} & h_{12} & h_{13} \\
h_{21} & h_{22} & h_{23} \\
h_{31} & h_{33} & h_{33}
\end{array}\right]^{i j}\left[\begin{array}{l}
0 \\
0 \\
1
\end{array}\right]=\left[\begin{array}{l}
0 \\
0 \\
0
\end{array}\right]
$$

and hence

$$
h_{k 3}^{i i}=-\sum_{\substack{j=1 \\ j \neq i}}^{j=n_{b n}} h_{k 3}^{i j}
$$

Second assume that $\stackrel{o}{\mathcal{F}} 1=1$ and $\stackrel{o}{\mathcal{F}}=\stackrel{o}{\mathcal{H}}=0$. Again, the logic of Remark 20 implies that no stresses and strains belong to the dual displacements

$$
\mathfrak{u}_{l}\left(M, Q_{i}\right)=\left[1|0| r_{2}\right]
$$

obtained from equation (99). Substitution of the above dual displacements into equation (141) yields

$$
\sum_{j=1}^{j=n_{b n}}\left[\begin{array}{lll}
h_{11} & h_{12} & h_{13} \\
h_{21} & h_{22} & h_{23} \\
h_{31} & h_{33} & h_{33}
\end{array}\right]^{i j}\left[\begin{array}{c}
1 \\
0 \\
r_{2}
\end{array}\right]^{j}=\left[\begin{array}{l}
0 \\
0 \\
0
\end{array}\right]
$$


from where

$$
h_{k 1}^{i i}=-\sum_{\substack{j=1 \\ j \neq i}}^{j=n_{b n}} h_{k 1}^{i j}-h_{k 3}^{i i} r_{2}^{i}-\sum_{\substack{j=1 \\ j \neq i}}^{j=n_{b n}} h_{k 3}^{i j} r_{2}^{j}=-\sum_{\substack{j=1 \\ j \neq i}}^{j=n_{b n}} h_{k 1}^{i j}-\sum_{\substack{j=1 \\ j \neq i}}^{j=n_{b n}} h_{k 3}^{i j}\left(r_{2}^{i}-r_{2}^{j}\right)
$$

Third we assume that $\stackrel{o}{\mathcal{F}}_{1}=\stackrel{o}{\mathcal{H}}=0$ and $\stackrel{o}{\mathcal{F}}_{2}=1$. Then repeating the logic leading to (146) we get

$$
h_{k 2}^{i i}=-\sum_{\substack{j=1 \\ j \neq i}}^{j=n_{b n}} h_{k 2}^{i j}-\sum_{\substack{j=1 \\ j \neq i}}^{j=n_{b n}} h_{k 3}^{i j}\left(r_{1}^{i}-r_{1}^{j}\right)
$$

Using equations (145), (146) and (147) we can avoid computing strongly singular integrals.

For an outer region, we have to add a further term to the equation system to be solved. Define the matrix $\tilde{\mathbf{u}}$ as

$$
\tilde{\mathbf{u}}^{T}=[\underbrace{\tilde{\mathfrak{u}}_{1}^{1} \tilde{\mathfrak{u}}_{2}^{1} \tilde{\mathfrak{u}}_{3}^{1}}_{\tilde{\mathbf{u}}_{1}^{T}}|\underbrace{\tilde{\mathfrak{u}}_{1}^{2} \tilde{\mathfrak{u}}_{2}^{2} \tilde{\mathfrak{u}}_{3}^{2}}_{\tilde{\mathbf{u}}_{2}^{T}}| \cdots \mid \underbrace{\tilde{\mathfrak{u}}_{1}^{n_{b n}} \tilde{\mathfrak{u}}_{2}^{n_{b n}} \tilde{\mathfrak{u}}_{3}^{n_{b n}}}_{\tilde{\mathbf{u}}_{n_{b n}}^{T}}]
$$

where the matrix $\tilde{\mathbf{u}}_{j}$ has the dual displacements $\tilde{\mathfrak{u}}_{k}$ taken at the points $Q_{j}\left(j=1, \ldots, n_{b n}\right)$ as its elements. With this notation the equation system to be solved for the unknown nodal values assumes the form

$$
\mathbf{H u}=\tilde{\mathbf{u}}+\mathbf{B t} .
$$

The next question is how to compute the strongly singular integrals for exterior regions. Our procedure is as follows: (a) determine $h_{k l}^{i i}$ under the assumption that the region under consideration is the interior region $A^{+}$; (b) then apply the formula

$$
\underbrace{h_{k l}^{i i}}_{\text {for } A^{-}}=\underbrace{h_{k l}^{i i}}_{\text {for } A^{+}}+\delta_{k l}
$$

The proof is based on equation (124). The details are, however, omitted here.

\subsection{Examples}

We have solved two external boundary value problems. First we consider the coordinate plane with a circular hole (Figure 5.b.); second the coordinate plane with a rigid inclusion (Figure 5.c.).

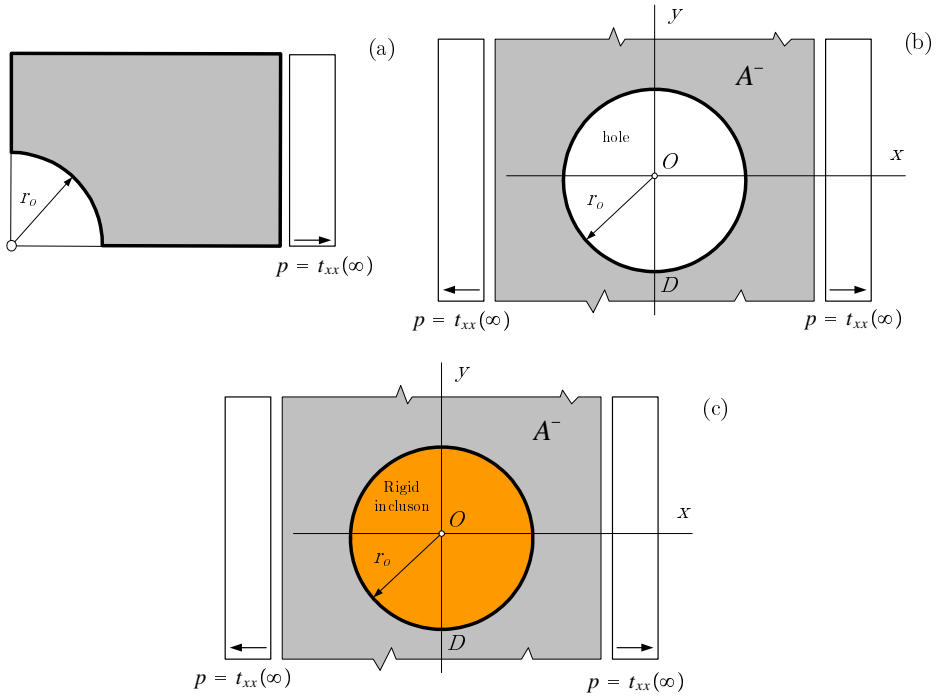

Figure 5: 
We remark that there exists an analytical solution for the stress concentration problem around the hole in an infinite plate of isotropic material if the stress state at infinity is $t_{x x}=p=$ constant $=100 \mathrm{n} / \mathrm{mm}^{2}$, $t_{x y}=t_{y x}=t_{y y}=\mu_{x z}=\mu_{y z}=0$, i.e., the plate is in tension [27, 28, 29]. The stress concentration factor $K$ for the above problem can be calculated from the following equations:

$$
K=\frac{t_{x x}\left(x=0, y=r_{o}\right)}{t_{x x}(\infty)}=\frac{3+F}{1+F}, \quad F=8(1-\nu) N^{2}\left[4+\frac{r_{o}^{2}}{l_{1}^{2}}+\frac{2 r_{o}}{l_{1}} \frac{K_{0}\left(r_{o} / l_{1}\right)}{K_{1}\left(r_{o} / l_{1}\right)}\right]^{-1}
$$

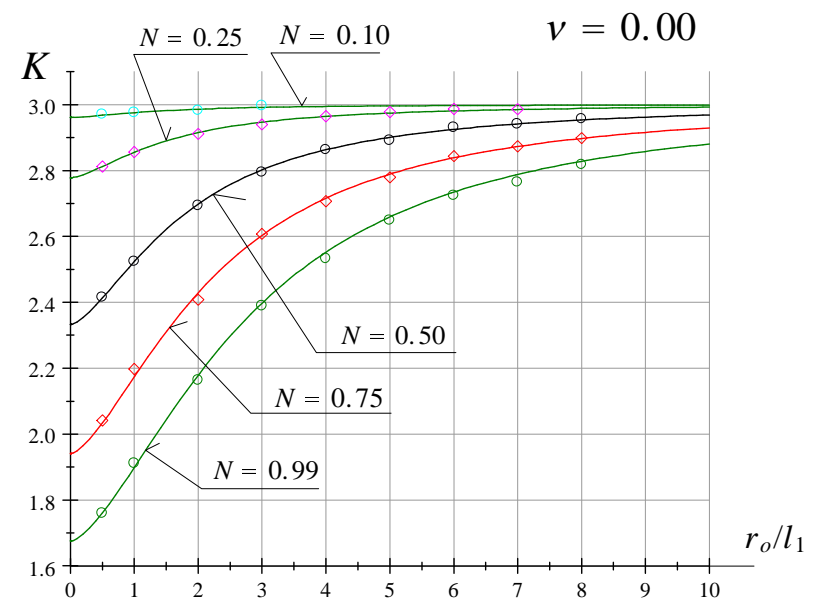

Figure 6:

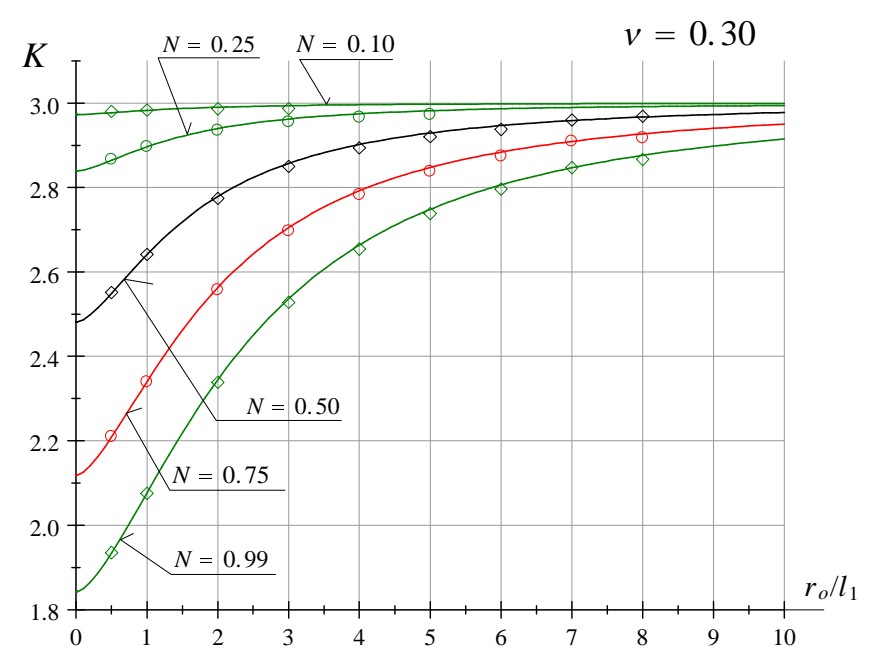

Figure 7:

We have carried out the computation under the assumption that $\mu=5 \mathrm{~N} / \mathrm{mm}^{2}, r_{o}=0.36 \mathrm{~mm}, \nu=0.0$ (see Figure 6) or $\nu=0.3$ (see Figure 7).

Figures 6 and 7 show the exact values for various couple numbers $N$ (solid lines) as well as the values computed. The latter are denoted by diamonds and circles. These fit well the graphs of the exact values.

Results for the rigid inclusion have been computed using the same data as for the circular hole, however there are no computational results for $\nu=0.0$. Figure 8 shows the normal stress $\sigma_{n}=\sigma_{r}$ against the polar angel $\theta \in[0, \pi / 2]$. The black line represents the classical solution. This curve fits very well the results computed for $N=0.01$. The graphs for $N=0.50, N=0.75$ and for $N=0.99$ (couple stress theory) are also depicted in Figure 8. 


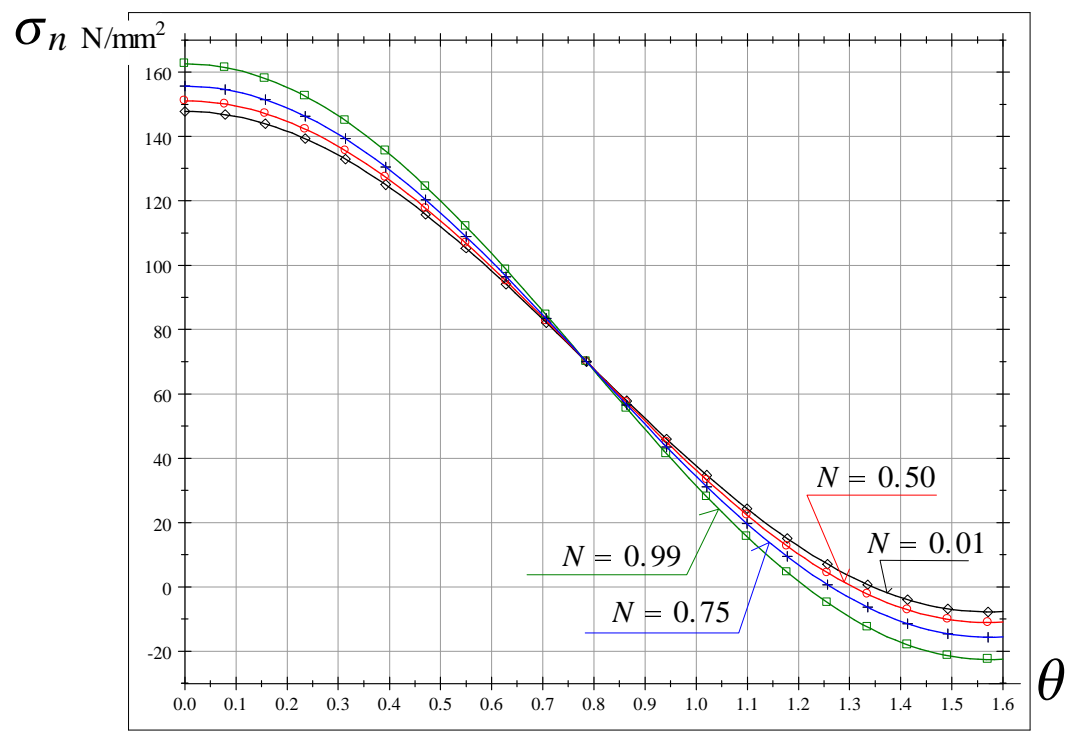

Figure 8:

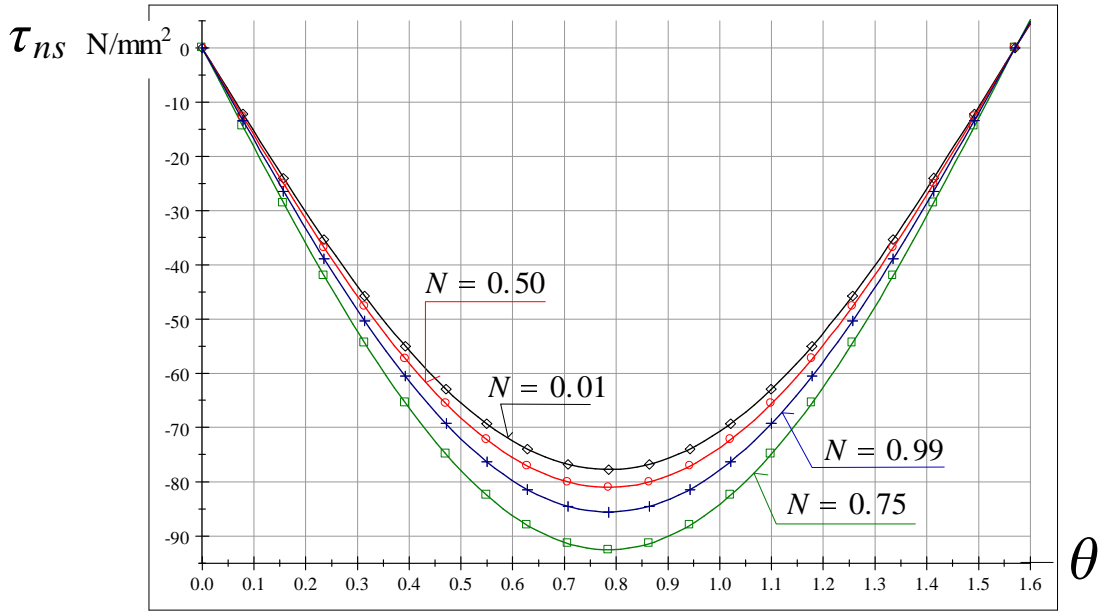

Figure 9:

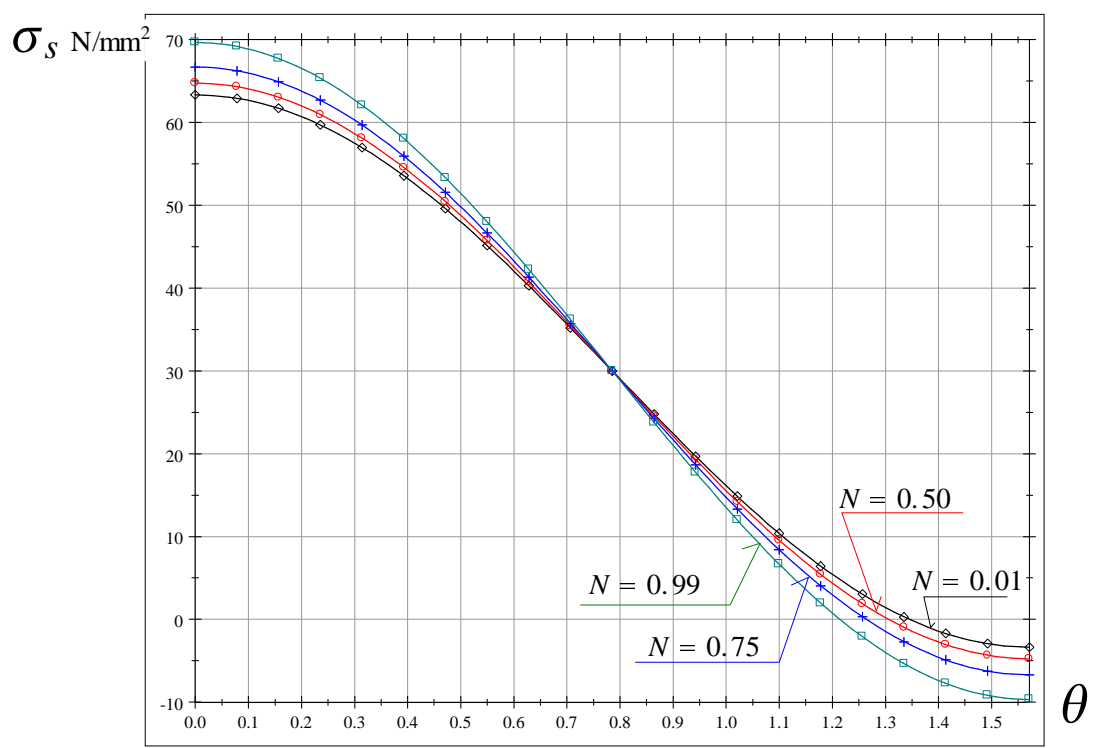

Figure 10: 
Figures 9 and 10 show the shear stress $\tau_{n s}=\tau_{r \theta}$ and the hoop stress $\sigma_{s}=\sigma_{\theta}$. The agreement with the classical solution for $N=0.01$ is again very good.

\section{Concluding remarks}

The present paper has dealt with the following issues:

1. Assuming isotropic and homogenous material we have presented the field equations and boundary conditions for the first plane problem of micropolar elasticity in a dual formulation. We have also clarified the supplementary conditions of single valuedness that strains should meet for a class of mixed boundary value problems both for simply and multiply connected regions.

2. By applying Galorkin functions and following the procedure presented in Kupradze's book [30] and elsewhere, we have derived the dual fundamental solutions of order one and two for the first plane problem of micropolar elasticity. ${ }^{1}$

3. We have set up the dual SomigLianA relations both for inner and for exterior regions. A constant stress state at infinity is a part of our formulation for exterior regions. We also developed an integral representation for the stresses.

4. We developed a solution algorithm, coded it in Fortran 90, and used it to numerically solve simple boundary value problems in order to demonstrate the applicability of the solution algorithm.

We remark that the supplementary conditions of single valuedness should be incorporated into the algorithm if (a) the number of arcs on which tractions are prescribed is more than one or (b) if in addition to this the region under consideration is multiply connected. Work on these issues is in progress.

\section{Acknowledgement}

The support provided by the Hungarian National Research Foundation within the framework of the project OTKA T 046834 is gratefully acknowledged.

\section{A Some matrices in the formulae for the stresses at internal points}

If we compare equations (67), (68) and (69) then we obtain

$$
\begin{gathered}
\mathcal{S}_{\beta 1}(M, Q)=\frac{a}{2 \pi k^{2}} \frac{1}{R^{2}}\left\{\frac{1}{2}\left(\delta_{\beta 1} r_{2}-\delta_{\beta 2} r_{1}+2 \frac{r_{\beta} r_{1} r_{2}}{R^{2}}\right)-\right. \\
\left.-\mathfrak{a} k^{2}\left(\delta_{\beta 1} r_{2}+\delta_{\beta 2} r_{1}-4 \frac{r_{\beta} r_{1} r_{2}}{R^{2}}\right) \mathcal{D}(k R)+\mathfrak{a} k^{2} \frac{r_{\beta} r_{1} r_{2}}{R} k K_{1}(k R)\right\}, \\
\mathcal{S}_{31}(M, Q)=\frac{a}{2 \pi k^{2}}\left(\frac{1}{2} \ln R+\frac{1}{4}+\frac{r_{2} r_{2}}{2 R^{2}}\right), \\
\mathcal{S}_{\beta 2}(M, Q)=\frac{a}{2 \pi k^{2}} \frac{1}{R^{2}}\left\{\frac{1}{2}\left(-r_{\beta}+2 \frac{r_{\beta} r_{2} r_{2}}{R^{2}}\right)-\right. \\
\left.-\mathfrak{a} k^{2}\left(2 \delta_{\beta 2} r_{2}+r_{\beta}-4 \frac{r_{\beta} r_{2} r_{2}}{R^{2}}\right) \mathcal{D}(k R)+\mathfrak{a} k^{2} \frac{r_{\beta} r_{2} r_{2}}{R} k K_{1}(k R)\right\},
\end{gathered}
$$

${ }^{1}$ This procedure is attributed to HöRMANDER $[23,1964]$ by the boundary element community. To our knowledge however it is A. I. LURIE who applied this technique first to determine the fundamental solutions for the 3D problems of classical elasticity [22, 1937] 


$$
\mathcal{S}_{32}(M, Q)=\frac{a}{2 \pi k^{2}} \frac{r_{1} r_{2}}{2 R^{2}},
$$

$$
\begin{gathered}
\mathcal{S}_{\beta 3}(M, Q)=\frac{a}{2 \pi k^{2}} \frac{1}{R^{2}}\left\{\frac{1}{2}\left(-r_{\beta}+2 \frac{r_{\beta} r_{1} r_{1}}{R^{2}}\right)-\right. \\
\left.-\mathfrak{a} k^{2}\left(2 \delta_{\beta 1} r_{1}+r_{\beta}-4 \frac{r_{\beta} r_{1} r_{1}}{R^{2}}\right) \mathcal{D}(k R)+\mathfrak{a} k^{2} \frac{r_{\beta} r_{1} r_{1}}{R} k K_{1}(k R)\right\}, \\
\mathcal{S}_{33}(M, Q)=\frac{a}{2 \pi k^{2}} \frac{r_{2} r_{1}}{2 R^{2}}, \\
\mathcal{S}_{\beta 4}(M, Q)=-\frac{a}{2 \pi k^{2}} \frac{1}{R^{2}}\left\{\frac{1}{2}\left(\delta_{2 \beta} r_{1}-\delta_{\beta 1} r_{2}+2 \frac{r_{\beta} r_{2} r_{1}}{R^{2}}\right)-\right. \\
\left.-\mathfrak{a} k^{2}\left(\delta_{2 \beta} r_{1}+\delta_{\beta 1} r_{2}-4 \frac{r_{\beta} r_{2} r_{1}}{R^{2}}\right) \mathcal{D}(k R)+\mathfrak{a} k^{2} \frac{r_{\beta} r_{2} r_{1}}{R} k K_{1}(k R)\right\} \\
\mathcal{S}_{34}(M, Q)=-\frac{a}{2 \pi k^{2}}\left[\frac{1}{2} \ln R+\frac{1}{4}+\frac{r_{1} r_{1}}{2 R^{2}}\right]
\end{gathered}
$$

and

$$
\begin{gathered}
\mathcal{M}_{\beta 1}(M, Q)=\frac{a}{2 \pi k^{2}}(-1)^{(\beta+1)}\left[\delta_{3-\beta, 2}\left(\frac{1}{2} \ln R+\frac{1}{4}\right)+\frac{r_{3-\beta} r_{2}}{2 R^{2}}\right] \\
\mathcal{M}_{31}(M, Q)=-\frac{a}{2 \pi k^{2}}\left[r_{2}\left(\frac{1}{2} \ln R+\frac{1}{4}\right)-\mathfrak{a} \frac{r_{2}}{R^{2}}\right] \\
\mathcal{M}_{\beta 2}(M, Q)=\frac{a}{2 \pi k^{2}}(-1)^{(\beta)}\left[\delta_{3-\beta, 1}\left(\frac{1}{2} \ln R+\frac{1}{4}\right)+\frac{r_{3-\beta} r_{1}}{2 R^{2}}\right] \\
\mathcal{M}_{32}(M, Q)=\frac{a}{2 \pi k^{2}}\left[r_{1}\left(\frac{1}{2} \ln R+\frac{1}{4}\right)-\mathfrak{a} \frac{r_{1}}{R^{2}}\right]
\end{gathered}
$$

If we recall that

$$
\stackrel{M}{\partial}(\ldots)=\stackrel{Q}{\partial}(\ldots)
$$

and compare equations (63), (67) and (106) then we can conclude that

$$
\begin{aligned}
S_{1 \lambda}(M, Q)=-\mathcal{S}_{\lambda 1}(M, Q), & S_{13}(M, Q)=\mathcal{S}_{31}(M, Q), \\
S_{2 \lambda}(M, Q)=-\mathcal{S}_{\lambda 2}(M, Q), & S_{23}(M, Q)=\mathcal{S}_{32}(M, Q), \\
S_{3 \lambda}(M, Q)=-\mathcal{S}_{\lambda 3}(M, Q), & S_{33}(M, Q)=\mathcal{S}_{33}(M, Q), \\
S_{4 \lambda}(M, Q)=-\mathcal{S}_{\lambda 4}(M, Q), & S_{43}(M, Q)=\mathcal{S}_{34}(M, Q)
\end{aligned}
$$

and

$$
\begin{gathered}
M_{1 \lambda}(M, Q)=\mathcal{M}_{\lambda 1}(M, Q), \quad M_{13}(M, Q)=-\mathcal{M}_{31}(M, Q) \\
M_{2 \lambda}(M, Q)=-\mathcal{M}_{\lambda 2}(M, Q), \quad M_{23}(M, Q)=-\mathcal{M}_{32}(M, Q) .
\end{gathered}
$$




\section{References}

[1] E. Cosserat and F. Cosserat. Théorie des corps déformables. Herman, Paris, 1979.

[2] A. C. Eringen and E. S. Suhubi. Nonlinear theory of simple microelastic solids i. International Journal of Engineering Sciences, (6), 1964.

[3] A. C. Eringen and E. S. Suhubi. Nonlinear theory of simple microelastic solids ii. International Journal of Engineering Sciences, (6), 1964.

[4] A. C. Eringen. Linear theory of micropolar elasticity. Journal of Mathematics and Mechanics, 15(6), 1967.

[5] W. Nowaczky. Theory of Micropolar Elasticity. Springer Verlag, Wien - NewYork - Udine, 1970.

[6] W. Nowaczky. Theory of Elasticity. Mir, Moscow, 1970. in Russian.

[7] E. Tonti. Variational Principles in Elastostatics. Meccanica, 14:201-208, 1967.

[8] E. Tonti. A Mathematical Model for Physical Theories I. II. Rendiconti Accademia Nazionale dei Lincei, pages $175-181 ; 351-356,1972$.

[9] Gy. Szeidl. Variational Principles and Solutions to Some Boundary Value Problems in the Asymmetric Elasticity [A nemszimmetrikus rugalmasságtan duál variációs elvei és egyes peremértékfeladatainak megoldása]. $\mathrm{Ph}$. D. Thesis, Hungarian Academy of Sciences, 1985. (in Hungarian).

[10] I. Kozák and Gy. Szeidl. The Field Equations and the Boundary Conditions with Force-Stresses and CoupleStresses in the Linearized Theory of Micropolar Elastostatics. Acta Techn. Hung., 90(3-4):57-80, 1980.

[11] H. Schaefer. Die Spannungsfunktionen des dreidimensionalen Kontinuums und des elastischen Körpers. $Z$. Angew. Math. Mech., 33:356-362, 1953.

[12] H. Schaefer. Die Spannungsfunktionen eines Kontinuums mit momentenspannungen i.-ii. Bulletin de l'Academie Polonaise des Sciences, Serie des sinces techniques, 15(1):63-67, 69-73, 1967.

[13] D. E. Carlson. On Günthers Stress Functions for Couple Stresses. Quart. Appl. Math., 25:139-146, 1967.

[14] W. Nowaczky. The Plane Problem of Micropolar Thermoelasticity. Archives of Mechanics, 22:3-26, 1970.

[15] D. Iesan. Existence Theorems in the Theory of Micropolar Elasticity. International Journal of Engineering Sciences, 8:777-791, 1970.

[16] P. Schiavone. Integral Equation Methods in Plane Asymmetric Elasticity. Journal of Elasticity, 43:31-43, 1996.

[17] Fuang-Yuan Keo and Keo Zoo Liang. Boundary element analysis of stress concentration in micropolar elastic plate. International Journal for Numerical Methods in Engineering, 40:1611-1622, 1997.

[18] Gy. Szeidl. Dual Variational Principles for the First Plane Problem of Micropolar Elastostatics. Publ TUHI., Series D, Natural Sciences, 35(3):3-20, 1982.

[19] Gy. Szeidl and I. Iván. Fundamental solutions and boundary integral equations for the first plane problem of micropolar elastostatics in dual system. In Conference on Numerical Methods and Computational Mechanics, Abstracts, page 74. University of Miskolc, July 15-19,1996.

[20] Gy. Szeidl and I. Iván. Macro Conditions of Compatibility and Strain Boundary Condititons for Some Mixed Plane Boundary Value Problems of Micropolar Elastostatics. Publications of the University of Miskolc, Series D, Natural Sciences, Mathematics, 36(2):35-45, 1996.

[21] I. Kozák and Gy. Szeidl. Contribution to the Field Equations and Boundary Conditions in Terms of Stresses of the First Plane Problem of Micropolar Elasticity. Publ TUHI., Series D, Natural Sciences, 34(2):135-146, 1981.

[22] A. I. Lurie. On Theory of Systems of Linear Differential Equations with Constant Ceofficients. Transactions of the Leningrad Industrial Institute, Number 6., Section of Physics and Mathematics, 6(3):31-36, 1937.

[23] L. Hörmander. Liner Partial Differential Operators. Springer-Verlag, Berlin, 1964.

[24] A. Erdélyi. Higher Transcendental Functions, volume 2. McGraw-Hill, NewYork-Toronto-London, 1953. 
[25] Janke-Emde-Lösch. Taflen Höheren Funktionen. B. G. Teubner Verlagsgesellschaft, Stuttgart, 1960.

[26] M. A. Jaswon and G. T. Symm. Integral Equation Methods in Potential Theory and Elastostatics. Academic Press, London - NewYork - San Francisco, 1977.

[27] Martin H. Sadd. Elasticity, Theory, Applications and Numerics. Elsevier, 2005. pp. 395-397.

[28] P. N. Kaloni and T. Ariman. Stress concentration effects in micropolar elasticity. J. Appl. Math. Phys., 18:136-141, 1967.

[29] P. N. Kaloni and T. Ariman. Stress concentration in micropolar elasticity. J. Appl. Math. Phys., 18:289-296, 1973.

[30] V. D. Kupradze. Potential Methods in Elasticity. State Publishing House, Series Physico-Mathematical Library, Moscow, 1963. (in Russian). 